\title{
The Extended Relativity Theory in Born-Clifford Phase Spaces with a Lower and Upper Length Scales and Clifford Group Geometric Unification
}

\author{
Carlos Castro
}

September 30, 2004

Center for Theoretical Studies of Physical Systems, Clark Atlanta University, Atlanta, GA. 30314, USA

\begin{abstract}
We construct the Extended Relativity Theory in Born-Clifford-Phase spaces with an upper and lower length scales (infrared/ultraviolet cutoff ). The invariance symmetry leads naturally to the real Clifford algebra $C l(2,6, R)$ and complexified Clifford $\mathrm{Cl}_{C}(4)$ algebra related to Twistors. We proceed with an extensive review of Smith's $8 D$ model based on the Clifford algebra $C l(1,7)$ that reproduces at low energies the physics of the Standard Model and Gravity; including the derivation of all the coupling constants, particle masses, mixing angles, ....with high precision. Further results by Smith are discussed pertaining the interplay among Clifford, Jordan, Division and Exceptional Lie algebras within the hierarchy of dimensions $D=26,27,28$ related to bosonic string, $M, F$ theory. Two Geometric actions are presented like the Clifford-Space extension of Maxwell's Electrodynamics, Brandt's action related the $8 D$ spacetime tangent-bundle involving coordinates and velocities ( Finsler geometries ) followed by a discussion ( based on results by Cho et al ) why Einstein's gravity in $m+n$ dimensions is equivalent to an $m$-dim Yang-Mills-like theory of diffemorphisms of an internal $n$-dim space which admits a holographic reduction to lower dimensions in the case of $A d S_{m} \times S^{n}$ and $d S_{m} \times H^{n}$ backgrounds. Finally we outline the reasons why a Clifford-Space Geometric Unification of all forces is a very reasonable avenue to consider and propose an Einstein-Hilbert type action in Clifford-Phase spaces (associated with the $8 D$ Phase space) as a Unified Field theory action candidate that should reproduce the physics of the Standard Model plus Gravity in the low energy limit.
\end{abstract}

\section{Introduction}

In recent years we have argued that the underlying fundamental physical principle behind string theory, not unlike the principle of equivalence and general covariance in Einstein's 
general relativity, might well be related to the existence of an invariant minimal length scale (Planck scale) attainable in nature. A scale relativistic theory involving spacetime resolutions was developed long ago by Nottale where the Planck scale was postulated as the minimum observer independent invariant resolution in Nature [2]. Since "points" cannot be observed physically with an ultimate resolution, they are fuzzy and smeared out into fuzzy balls of Planck radius of arbitrary dimension. For this reason one must construct a theory that includes all dimensions (and signatures) on the equal footing. Because the notion of dimension is a topological invariant, and the concept of a fixed dimension is lost due to the fuzzy nature of points, dimensions are resolution-dependent, one must also include a theory with all topologies as well. It turned out that Clifford algebras contained the appropriate algebro-geometric features to implement this principle of polydimensional transformations that reshuffle a five-brane history for a membrane history, for example. For an extensive review of this Extended Relativity Theory in Clifford Spaces that encompasses the unified dynamics of all p-branes, for different values of the dimensions of the extended objects, and numerous physical consequences, see [1]

A Clifford-space dynamical derivation of the stringy-minimal length uncertainty relations was furnished in [39]. The dynamical consequences of the minimal-length in Newtonian dynamics have been recently reviewed by [38]. The idea of minimal length ( the Planck scale $L_{P}$ ) can be incorporated within the context of the maximal acceleration Relativity principle [20] $a_{\max }=c^{2} / L_{P}$ in Finsler Geometries [12]. A different approach than the one based on Finsler Geometries is the pseudo-complex Lorentz group description by Schuller [13] related to the effects of maximal acceleration in Born-Infeld models that also maintains Lorentz invariance, in contrast to the approaches of Double Special Relativity (DSR) [22] where the Lorentz symmetry is deformed . Quantum group deformations of the Poincare symmetry and of Gravity have been analyzed by [21] where the deformation parameter $q$ could be interpreted in terms of an upper and lower scale as $q=e^{L_{P} / R}$ such that the undeformed limit $q=1$ can be attained when $L_{P} \rightarrow 0$ and/or when $R \rightarrow \infty$ [20]. For a discussions on the open problems of Double Special Relativity theories based on kappa-deformed Poincare symmetries [14] and motivated by the anomalous Lorentzviolating dispersion relations in the ultra high energy cosmic rays [23, 25], we refer to $[22]$.

An upper limit on the maximal acceleration of particles was proposed long ago by Cainello [8]. This idea is a direct consequence of a suggestion made years earlier by Max Born on a Dual Relativity principle operating in Phase Spaces [7], [26] where there is an upper bound on the four-force (maximal string tension or tidal forces in strings ) acting on a particle as well as an upper bound in the particle's velocity given by the speed of light. For a recent status of the geometries behind maximal-acceleration see [25]; its relation to the Double Special Relativity programs was studied by [11] and the possibility that Moyal deformations of Poincare algebras could be related to the kappa-deformed Poincare algebras was raised in [20]. A thorough study of Finsler geometry and Clifford algebras has been undertaken by Vacaru [?] where Clifford/spinor structures were defined with respect to Nonlinear connections associated with certain nonholonomic modifications of RiemannCartan gravity. The study of non-holonomic Clifford-Structures in the construction of a Noncommutative Riemann-Finsler Geometry has recently been advanced by [?]. 
Other implications of the maximal acceleration principle in Nature, like neutrino oscillations and other phenomena, have been studied by [10], [17], [18] Recently, the variations of the fine structure constant $\alpha$ [15] with the cosmological accelerated expansion of the Universe was recast as a renormalization group-like equation governing the cosmological reshift (Universe scale) variations of $\alpha$ based on this maximal acceleration principle in Nature [20]. The fine structure constant was smaller in the past. Pushing the cuttof scale to the minimum Planck scale led to the intriguing result that the fine structure constant could have been extremely small (zero) in the early Universe and that all matter in the Universe could have emerged via the Unruh-Rindler-Hawking effect (creation of radiation/matter) due to the acceleration w.r.t the vacuum frame of reference. For reviews on the alledged variations of the fundamental constants in Nature see [16] .

The outline of this work goes as follows. In section 2.1 we review the Dual Phase Space Relativity and show why the Planck areas are invariant under acceleration-boosts transformations.

In 2.2 we investigate the consequences of the Mach's principle of inertia within the context of the Dual Phase Space Relativity Principle which is compatible with the EddingtonDirac large numbers coincidence and may provide with a very plausible physical reason behind the observed anomalous Pioneer acceleration [3] and a solution to the riddle of the cosmological constant problem [2] . The cosmological implications of Non-Archimedean Geometry [78] by assigning an upper impassible scale in Nature [2] and the cosmological variations of the fundamental constants are also discussed.

In 3 we review the Extended Relativity Theory in Clifford Spaces and some important applications, like superluminal propagation and modified dispersion relations in connection to the derivation of the minimal length stringy uncertainty relations.

In 4 we construct the Dual Extended Relativity Theory in Clifford-Phase spaces with an upper and lower length scales simultaneously ( infrared/ultraviolet cutoff ) ; study the symmetry transformations laws under velocity and acceleration ( force ) boosts ; explain the importance of the Clifford algebra $C l(2,6)$; the complexified Clifford algebras and Quaternions $\mathbf{H}$ and their association to ordinary twistors and their Quaternionic extensions.

We finalize section 4 by studying the modified Newtonian dynamics based on Yang's Noncommutative Spacetime algebra, involving a lower and upper scale [30] that has been revisited recently by us [29] in the context of holography and area-quantization in Cspaces ( Clifford spaces ) ; in the physics of $D$-branes and covariant Matrix models by [31] and within the context of Lie algebra stability by [42]. A different algebra with two length scales has been studied by [37] in order to account for modifications of Newtonian dynamics ( that also violates the equivalence principle).

In 5.1 we review extensively Smith's $8 D$ model [32] based on the Clifford algebra $\mathrm{Cl}(1.7)$ that reproduces the physics of the Standard Model and Gravity, including the derivation of the coupling constants, particle masses, mixing angles, .... with high precision. In 5.2 we continue by discussing further results by [32] pertaining the interplay among Clifford, Jordan, Division and Exceptional Lie algebras within the chain of dimensions $D=26,27,28$ related to bosonic string, M, F theory.

In 6 we analyze different geometric actions, like the Clifford-space extensions of 
Maxwell's EM; Brandt's action [12] related the 8D spacetime tangent-bundle involving coordinates and velocities ( Finsler geometries ) and, finally, we discuss in further detail why Einstein's gravity in $m+n$ dimensions is equivalent to an $m$-dim Yang-Mills-like theory of diffemorphisms of an internal $n$-dim space which admits a holographic reduction to lower dimensions in the case of $A d S_{m} \times S^{n}$ and $d S_{m} \times H^{n}$ backgrounds.

Finally in 7 we outline the reasons why a Clifford-Space Geometric Unification of all forces is a reasonable avenue to take and propose an Einstein-Hilbert type action in the Clifford-Phase space (associated with the 8D Phase space), as the Unified Field theory action that should reduce to the Standard Model plus Gravity in the low energy limit.

\section{Dual Phase-Space Relativity}

In this section we will review in detail the Born's Dual Phase Space Relativity and the principle of Maximal-acceleration Relativity [20] from the perspective of $8 D$ Phase Spaces and the $U(1,3)$ Group. The $U(1,3)=S U(1,3) \otimes U(1)$ Group transformations, which leave invariant the phase-space intervals under rotations, velocity and acceleration boosts, were found by Low [26] and can be simplified drastically when the velocity/acceleration boosts are taken to lie in the $z$-direction, leaving the transverse directions $x, y, p_{x}, p_{y}$ intact ; i.e., the $U(1,1)=S U(1,1) \otimes U(1)$ subgroup transformations that leave invariant the phase-space interval are given by (in units of $\hbar=c=1$ )

$$
\begin{gathered}
(d \omega)^{2}=(d T)^{2}-(d X)^{2}+\frac{(d E)^{2}-(d P)^{2}}{b^{2}}= \\
(d \tau)^{2}\left[1+\frac{(d E / d \tau)^{2}-(d P / d \tau)^{2}}{b^{2}}\right]=(d \tau)^{2}\left[1-\frac{m^{2} g^{2}(\tau)}{m_{P}^{2} A_{\max }^{2}}\right] .
\end{gathered}
$$

where we have factored out the proper time infinitesimal $(d \tau)^{2}=d T^{2}-d X^{2}$ in eq- $(2-1)$ and the maximal proper-force is set to be $b \equiv m_{P} A_{\max } \cdot m_{P}$ is the Planck mass $1 / L_{P}$ so that $b=\left(1 / L_{P}\right)^{2}$, may also be interpreted as the maximal string tension when $L_{P}$ is the Planck scale.

The quantity $g(\tau)$ is the proper four-acceleration of a particle of mass $m$ in the $z$ direction which we take to be defined by the $X$ coordinate. The interval $(d \omega)^{2}$ described by Low $[26]$ is $U(1,3)$-invariant for the most general transformations in the $8 D$ phase-space. These transformations are rather elaborate, so we refer to the references [26] for details. The appearance of the $U(1,3)$ group in $8 D$ Phase Space is not too surprising since it could be seen as the " complex doubling " version of the Lorentz group $S O(1,3)$. Low discussed the irreducible unitary representations of such $U(1,3)$ group and the relevance for the strong interactions of quarks and hadrons since $U(1,3)$, with 16 generators, contains the $S U(3)$ group.

The analog of the Lorentz relativistic factor in eq-(2-1) involves the ratios of two proper forces. One variable force is given by $m g(\tau)$ and the maximal proper force sustained by an elementary particle of mass $m_{P}$ (a Planckton) is assumed to be $F_{\max }=m_{\text {Planck }} c^{2} / L_{P}$. 
When $m=m_{P}$, the ratio-squared of the forces appearing in the relativistic factor of eq(2-1 ) becomes then $g^{2} / A_{\max }^{2}$, and the phase space interval coincides with the geometric interval discussed by [13], [10], [17], [18] .

The transformations laws of the coordinates in that leave invariant the interval (2-1) were given by [26]:

$$
\begin{array}{ll}
T^{\prime}=T \cosh \xi+\left(\frac{\xi_{v} X}{c^{2}}+\frac{\xi_{a} P}{b^{2}}\right) \frac{\sinh \xi}{\xi} . & 2-2 a \\
E^{\prime}=E \cosh \xi+\left(-\xi_{a} X+\xi_{v} P\right) \frac{\sinh \xi}{\xi} . & 2-2 b \\
X^{\prime}=X \cosh \xi+\left(\xi_{v} T-\frac{\xi_{a} E}{b^{2}}\right) \frac{\sinh \xi}{\xi} . & 2-2 c \\
P^{\prime}=P \cosh \xi+\left(\frac{\xi_{v} E}{c^{2}}+\xi_{a} T\right) \frac{\sinh \xi}{\xi} . & 2-2 d
\end{array}
$$

The $\xi_{v}$ is velocity-boost rapidity parameter and the $\xi_{a}$ is the force/acceleration-boost rapidity parameter of the primed-reference frame. They are defined respectively :

$$
\tanh \left(\frac{\xi_{v}}{c}\right)=\frac{v}{c} . \quad \tanh \left(\frac{\xi_{a}}{b}\right)=\frac{m a}{m_{P} A_{\max }} . \quad 2-3
$$

The effective boost parameter $\xi$ of the $U(1,1)$ subgroup transformations appearing in eqs- $(2-2 \mathrm{a}, 2-2 \mathrm{~d})$ is defined in terms of the velocity and acceleration boosts parameters $\xi_{v}, \xi_{a}$ respectively as:

$$
\xi \equiv \sqrt{\frac{\xi_{v}^{2}}{c^{2}}+\frac{\xi_{a}^{2}}{b^{2}}}
$$

Our definition of the rapidity parameters are different than those in [26].

Straightforward algebra allows us to verify that these transformations leave the interval of eq- (2-1) in classical phase space invariant. They are are fully consistent with Born's duality Relativity symmetry principle $[7](Q, P) \rightarrow(P,-Q)$. By inspection we can see that under Born duality, the transformations in eqs- $(2-2 \mathrm{a}, 2-2 \mathrm{~d})$ are rotated into each other, up to numerical $b$ factors in order to match units. When on sets $\xi_{a}=0$ in (22a, 2-2d) one recovers automatically the standard Lorentz transformations for the $X, T$ and $E, P$ variables separately, leaving invariant the intervals $d T^{2}-d X^{2}=(d \tau)^{2}$ and $\left(d E^{2}-d P^{2}\right) / b^{2}$ separately.

When one sets $\xi_{v}=0$ we obtain the transformations rules of the events in Phase space, from one reference-frame into another uniformly-accelerated frame of reference, $a=$ constant, whose acceleration-rapidity parameter is in this particular case:

$$
\xi \equiv \frac{\xi_{a}}{b} . \quad \tanh (\xi)=\frac{m a}{m_{P} A_{\max }} .
$$

The transformations for pure acceleration-boosts in Phase Space are:

$$
T^{\prime}=T \cosh \xi+\frac{P}{b} \sinh \xi . \quad 2-6 a
$$




$$
\begin{array}{rlrl}
E^{\prime} & =E \cosh \xi-b X \sinh \xi . & 2-6 b \\
X^{\prime}=X \cosh \xi-\frac{E}{b} \sinh \xi . & 2-6 c \\
P^{\prime}=P \cosh \xi+b T \sinh \xi . & 2-6 d
\end{array}
$$

It is straightforwad to verify that the transformations (2-6a, 2-6c) leave invariant the fully phase space interval (2-1) but does not leave invariant the proper time interval $(d \tau)^{2}=d T^{2}-d X^{2}$. Only the combination:

$$
(d \omega)^{2}=(d \tau)^{2}\left(1-\frac{m^{2} g^{2}}{m_{P}^{2} A_{\max }^{2}}\right) \quad 2-7
$$

is truly left invariant under pure acceleration-boosts in Phase Space. Once again, can verify as well that these transformations satisfy Born's duality symmetry principle:

$$
(T, X) \rightarrow(E, P) .(E, P) \rightarrow(-T,-X) . \quad 2-7
$$

and $b \rightarrow \frac{1}{b}$. The latter Born duality transformation is nothing but a manifestation of the large/small tension duality principle reminiscent of the $T$-duality symmetry in string theory; i.e. namely, a small/large radius duality, a winding modes/ Kaluza-Klein modes duality symmetry in string compactifications and the Ultraviolet/Infrared entanglement in Noncommutative Field Theories. Hence, Born's duality principle in exchanging coordinates for momenta could be the underlying physical reason behind $T$-duality in string theory.

The composition of two succesive pure acceleration-boosts is another pure accelerationboost with acceleration rapidity given by $\xi^{\prime \prime}=\xi+\xi^{\prime}$. The addition of proper forces ( accelerations ) follows the usual relativistic composition rule:

$$
\tanh \xi^{\prime \prime}=\tanh \left(\xi+\xi^{\prime}\right)=\frac{\tanh \xi+\tanh \xi^{\prime}}{1+\tanh \xi \tanh \xi^{\prime}} \Rightarrow \frac{m a^{\prime \prime}}{m_{P} A}=\frac{\frac{m a}{m_{P} A}+\frac{m a^{\prime}}{m_{P} A}}{1+\frac{m^{2} a a^{\prime}}{m_{P}^{2} A^{2}}} . \quad 2-8
$$

and in this fashion the upper limiting proper acceleration is never surpassed like it happens with the ordinary Special Relativistic addition of velocities.

The group properties of the full combination of velocity and acceleration boosts eqs(2-2a, 2-2d) in Phase Space requires much more algebra [20]. A careful study reveals that the composition rule of two succesive full transformations is given by $\xi^{\prime \prime}=\xi+\xi^{\prime}$ and the transformation laws are preserved if, and only if, the $\xi ; \xi^{\prime} ; \xi^{\prime \prime} \ldots .$. parameters obeyed the suitable relations:

$$
\begin{aligned}
\frac{\xi_{a}}{\xi}=\frac{\xi_{a}^{\prime}}{\xi^{\prime}}=\frac{\xi_{a}^{\prime \prime}}{\xi^{\prime \prime}}=\frac{\xi_{a}^{\prime \prime}}{\xi+\xi^{\prime}} . & 2-9 a \\
\frac{\xi_{v}}{\xi}=\frac{\xi_{v}^{\prime}}{\xi^{\prime}}=\frac{\xi_{v}^{\prime \prime}}{\xi^{\prime \prime}}=\frac{\xi_{v}^{\prime \prime}}{\xi+\xi^{\prime}} & 2-9 b
\end{aligned}
$$


Finally we arrive at the compostion law for the effective, velocity and acceleration boosts parameters $\xi^{\prime \prime} ; \xi_{v}^{\prime \prime} ; \xi_{a}^{\prime \prime}$ respectively:

$$
\begin{array}{ll}
\xi_{v}^{\prime \prime}=\xi_{v}+\xi_{v}^{\prime} . & 2-10 a \\
\xi_{a}^{\prime \prime}=\xi_{a}+\xi_{a}^{\prime} . & 2-10 b \\
\xi^{\prime \prime}=\xi+\xi^{\prime} . & 2-10 c
\end{array}
$$

The above relations among the parameters are required in order to prove the $U(1,1)$ group composition law of the transformations in order to have a truly Maximal-Acceleration Phase Space Relativity theory resulting from a Phase-Space change of coordinates in the cotangent bundle of spacetime.

\subsection{Planck-Scale Areas are Invariant under Acceleration Boosts}

Having displayed explicity the Group transformations rules of the coordinates in Phase space we will show why infinite acceleration-boosts (which is not the same as infinite proper acceleration) preserve Planck-Scale Areas [20] as a result of the fact that $b=$ $\left(1 / L_{P}^{2}\right)$ equals the maximal invariant force, or string tension, if the units of $\hbar=c=1$ are used.

At Planck-scale $L_{P}$ intervals/increments in one reference frame we have by definition (in units of $\hbar=c=1$ ): $\Delta X=\Delta T=L_{P}$ and $\Delta E=\Delta P=\frac{1}{L_{P}}$ where $b \equiv \frac{1}{L_{P}^{2}}$ is the maximal tension. From eqs- $(2-6 \mathrm{a}, 2-6 \mathrm{~d})$ we get for the transformation rules of the finite intervals $\Delta X, \Delta T, \Delta E, \Delta P$, from one reference frame into another frame, in the infinite acceleration-boost limit $\xi \rightarrow \infty$,

$$
\begin{gathered}
\Delta T^{\prime}=L_{P}(\cosh \xi+\sinh \xi) \rightarrow \infty \\
\Delta E^{\prime}=\frac{1}{L_{P}}(\cosh \xi-\sinh \xi) \rightarrow 0
\end{gathered}
$$

by a simple use of L'Hopital's rule or by noticing that both $\cosh \xi ; \sinh \xi$ functions approach infinity at the same rate.

$$
\begin{array}{ll}
\Delta X^{\prime}=L_{P}(\cosh \xi-\sinh \xi) \rightarrow 0 . & 2-11 c \\
\Delta P^{\prime}=\frac{1}{L_{P}}(\cosh \xi+\sinh \xi) \rightarrow \infty & 2-11 d
\end{array}
$$

where the discrete displacements of two events in Phase Space are defined: $\Delta X=X_{2}-$ $X_{1}=L_{P}, \Delta E=E_{2}-E_{1}=\frac{1}{L_{P}}, \Delta T=T_{2}-T_{1}=L_{P}$ and $\Delta P=P_{2}-P_{1}=\frac{1}{L_{P}}$.

Due to the identity:

$$
(\cosh \xi+\sinh \xi)(\cosh \xi-\sinh \xi)=\cosh ^{2} \xi-\sinh ^{2} \xi=1
$$


one can see from eqs-(2-11a, 2-11d) that the Planck-scale Areas are truly invariant under infinite acceleration-boosts $\xi=\infty$ :

$$
\begin{array}{ll}
\Delta X^{\prime} \Delta P^{\prime}=0 \times \infty=\Delta X \Delta P\left(\cosh ^{2} \xi-\sinh ^{2} \xi\right)=\Delta X \Delta P=\frac{L_{P}}{L_{P}}=1 . & 2-13 a \\
\Delta T^{\prime} \Delta E^{\prime}=\infty \times 0=\Delta T \Delta E\left(\cosh ^{2} \xi-\sinh ^{2} \xi\right)=\Delta T \Delta E=\frac{L_{P}}{L_{P}}=1 . & 2-13 b \\
\Delta X^{\prime} \Delta T^{\prime}=0 \times \infty=\Delta X \Delta T\left(\cosh ^{2} \xi-\sinh ^{2} \xi\right)=\Delta X \Delta T=\left(L_{P}\right)^{2} . & 2-13 c \\
\Delta P^{\prime} \Delta E^{\prime}=\infty \times 0=\Delta P \Delta E\left(\cosh ^{2} \xi-\sinh ^{2} \xi\right)=\Delta P \Delta E=\frac{1}{L_{P}^{2}} . & 2-13 d
\end{array}
$$

It is important to emphasize that the invariance property of the minimal Planck-scale Areas (maximal Tension) is not an exclusive property of infinite acceleration boosts $\xi=\infty$, but, as a result of the identity $\cosh ^{2} \xi-\sinh ^{2} \xi=1$, for all values of $\xi$, the minimal Planck-scale Areas are always invariant under any acceleration-boosts transformations. Meaning physically, in units of $\hbar=c=1$, that the Maximal Tension (or maximal Force) $b=\frac{1}{L_{P}^{2}}$ is a true physical invariant universal quantity. Also we notice that the Phasespace areas, or cells, in units of $\hbar$, are also invariant! The pure-acceleration boosts transformations are "symplectic". It can be shown also that areas greater ( smaller ) than the Planck-area remain greater ( smaller ) than the invariant Planck-area under acceleration-boosts transformations.

The infinite acceleration-boosts are closely related to the infinite red-shift effects when light signals barely escape Black hole Horizons reaching an asymptotic observer with an infinite redshift factor. The important fact is that the Planck-scale Areas are truly maintained invariant under acceleration-boosts. This could reveal very important information about Black-holes Entropy and Holography.

\subsection{The Machian Principle and Eddington-Dirac Large Num- bers Coincidence}

A natural action associated with the invariant interval in Phase-Space given by eq-(2-1) is :

$$
S=m \int d \tau \sqrt{1+\frac{m^{2}}{m_{P}^{2} a^{2}}\left(d^{2} x^{\mu} / d \tau^{2}\right)\left(d^{2} x_{\mu} / d \tau^{2}\right)} .
$$

The proper-acceleration is orthogonal to the proper-velocity and this can be easily verified by differentiating the timelike proper-velocity squared:

$$
V^{2}=\frac{d x^{\mu}}{d \tau} \frac{d x_{\mu}}{d \tau}=V^{\mu} V_{\mu}=1>0 \Rightarrow \frac{d V^{\mu}}{d \tau} V_{\mu}=\frac{d^{2} x^{\mu}}{d \tau^{2}} V_{\mu}=0 . \quad 2-15
$$


which implies that the proper-acceleration is spacelike:

$$
-g^{2}(\tau)=\frac{d^{2} x^{\mu}}{d \tau^{2}} \frac{d^{2} x_{\mu}}{d \tau^{2}}<0 \Rightarrow S=m \int d \tau \sqrt{1-\frac{m^{2} g^{2}}{m_{P}^{2} a^{2}}}=m \int d \omega . \quad 2-16
$$

where the analog of the Lorentz time-dilation factor in Phase-space is now given by

$$
d \omega=d \tau \sqrt{1-\frac{m^{2} g^{2}(\tau)}{m_{P}^{2} a^{2}}}
$$

namely,

$$
(d \omega)^{2}=\Omega^{2} d \tau^{2}=\left[1-\frac{m^{2} g^{2}(\tau)}{m_{P}^{2} a^{2}}\right] g_{\mu \nu} d x^{\mu} d x^{\nu}
$$

The invariant proper interval is no longer the standard proper-time $\tau$ but is given by the quantity $\omega(\tau)$. The deep connection between the physics of maximal acceleration and Finsler geometry has been analyzed by [12]. The action is real-valued if, and only if, $m^{2} g^{2}<m_{P}^{2} a^{2}$ in the same fashion that the action in Minkowski spacetime is real-valued if, and only if, $v^{2}<c^{2}$. This is the physical reason why there is an upper bound in the proper-four force acting on a fundamental particle given by $(m g)_{\text {bound }}=m_{P}\left(c^{2} / L_{P}\right)=m_{P}^{2}$ in natural units of $\hbar=c=1$.

The Eddington-Dirac large numbers coincidence ( and an ultraviolet/infrared entanglement ) can be easily implemented if one equates the upper bound on the proper-four force sustained by a fundamental particle, $(m g)_{b o u n d}=m_{P}\left(c^{2} / L_{P}\right)$, with the proper-four force associated with the mass of the (observed ) universe $M_{U}$, and whose minimal acceleration $c^{2} / R$ is given in terms of an infrared-cutoff $R$ ( the Hubble horizon radius ). Equating these proper-four forces gives

$$
\frac{m_{P} c^{2}}{L_{P}}=\frac{M_{U} c^{2}}{R} \Rightarrow \frac{M_{U}}{m_{P}}=\frac{R}{L_{P}} \sim 10^{61}
$$

from this equality of proper-four forces associated with a maximal/minimal acceleration one infers $M_{U} \sim 10^{61} m_{\text {Planck }} \sim 10^{61} 10^{19} m_{\text {proton }}=10^{80} m_{\text {proton }}$ which agrees with observations and with the Eddington-Dirac number $10^{80}$ :

$$
N=10^{80}=\left(10^{40}\right)^{2} \sim\left(\frac{F_{e}}{F_{G}}\right)^{2} \sim\left(\frac{R}{r_{e}}\right)^{2} .
$$

where $F_{e}=e^{2} / r^{2}$ is the electrostatic force between an electron and a proton ; $F_{G}=$ $G m_{e} m_{\text {proton }} / r^{2}$ is the corresponding gravitational force and $r_{e}=e^{2} / m_{e} \sim 10^{-13} \mathrm{~cm}$ is the classical electron radius ( in units $\hbar=c=1$ ).

One may notice that the above equation (3-5) is also consistent with the Machian postulate that the rest mass of a particle is determined via the gravitational potential energy due to the other masses in the universe. In particular, by equating :

$$
m_{i} c^{2}=G m_{i} \sum_{j} \frac{m_{j}}{\left|r_{i}-r_{j}\right|}=\frac{G m_{i} M_{U}}{R} \Rightarrow \frac{c^{2}}{G}=\frac{M_{U}}{R} .
$$


Due to the negative binding energy, the composite mass $m_{12}$ of a system of two objects of mass $m_{1}, m_{2}$ is not equal to the sum $m_{1}+m_{2}>m_{12}$. We can now arrive at the conclusion that the minimal acceleration $c^{2} / R$ is also the same acceleration induced on a test particle of mass $m$ by a spherical mass distribution $M_{U}$ inside a radius $R$. The acceleration felt by a test particle of mass $m$ sitting at the edge of the observable Universe ( at the Hubble horizon radius $R$ ) is:

$$
\frac{G M_{U}}{R^{2}}=a .
$$

From the last two equations one gets the same expression for the minimal acceleration :

$$
a=a_{\text {minimal }}=\frac{c^{2}}{R} \text {. }
$$

which is of the same order of magnitude as the anomalous acceleration of the Pioneer and Galileo spacecrafts $a \sim 10^{-8} \mathrm{~cm} / \mathrm{s}^{2}$. Nottale has invoked the Machian principle of intertia [3] adopting a local and global inertial coordinate system at the scale of the solar system in order to explain the origins of this Pioneer-Galileo anomalous constant acceleration.

Let us examine closer the equality between the proper-four forces

$$
\frac{m_{P} c^{2}}{L_{P}}=\frac{M_{U} c^{2}}{R} \Rightarrow \frac{m_{P}}{L_{P}}=\frac{M_{U}}{R}=\frac{c^{2}}{G} .
$$

The last term in eq-(2-24) is directly obtained after implementing the Machian principle. Thus, one concludes from eq-( 2-24) that as the universe evolves in time one must have the conserved ratio of the quantities $M_{U} / R=c^{2} / G=m_{P} / L_{P}$. This interesting possibility, advocated by Dirac long ago, for the fundamental constants $\hbar, c, G, \ldots$. to vary over cosmological time is a plausible idea with the provision that the above ratios satisfy the relations in eq-(2-10) at any given moment of cosmological time. If the fundamental constants do not vary over time then the ratio $M_{U} / R=c^{2} / G$ must refer then to the asymptotic values of the Hubble horizon radius $R=R_{\text {asymptotic. }}$. A related approach to the idea of an impassible upper asymptotic length $R$ has been advocated by Scale Relativity [2] and in [78] where a Cosmology based on non-Archimedean geometry was proposed by recurring to p-adic numbers. For example, a Non-Archimedean number addition law of two masses $m_{1}, m_{2}$ does not follow the naive addition rule $m_{1}+m_{2}$ but instead:

$$
m_{1} \bullet m_{2}=\frac{m_{1}+m_{2}}{1+\left(m_{1} m_{2} / M_{U}^{2}\right)} .
$$

which is similar to the composition law of velocities in ordinary Relativity in terms of the speed of light. When the masses $m_{1}, m_{2}$ are much smaller than the universe mass $M_{U}$ one recovers the ordinary addition law. Similar considerations follow in the Non-Archimedean compostion law of lengths such that the upper length $R_{\text {asym }}$ is never surpassed. For further references on p-adic numbers and Physics were refer to [34]. A Mersenne prime, $M_{p}=$ $2^{p}-1=$ prime, for $p=$ prime, $\mathrm{p}$-adic hierarchy of scales in Particle physics and Cosmology has been discussed by Pitkannen and Noyes where many of the the fundamental energy scales, masses and couplings in Physics has been obtained [35], [36]. For example, the 
Mersenne prime $M_{127}=2^{127}-1 \sim 10^{38} \sim\left(m_{\text {Planck }} / m_{\text {proton }}\right)^{2}$. The derivation of the Standard Model parameters from first principle has obtained by Smith [32] and Beck [33].

In [20] we proposed a plausible explanation of the variable fine structure constant phenomenon based on the maximal-acceleration relativity principle in phase-space by modifying the Robertson-Friedmann-Walker metric by a similar ( acceleration-dependent ) conformal factor. It led us to the conclusion that the universe could have emerged from the vacuum as a quantum bubble ( or " brane-world") of Planck mass and Planck radius that expanded ( w.r.t to the vacuum ) at the speed of light with a maximal acceleration $a=c^{2} / L_{p}$. Afterwards the acceleration began to slow down as matter was being created from the vacuum, via an Unruh-Rindler-Hawking effect, from this initial maximal value $c^{2} / L_{p}$ to the value of $c^{2} / R \sim 10^{-8} \mathrm{~cm} / \mathrm{s}^{2}$ (of the same order of magnitude as the Pioneer anomalous acceleration). Namely, as the universe expanded, matter was being created from the vacuum via the Unruh-Rindler-Hawking effect ( which must not to be confused with Hoyle's Steady State Cosmolgy ) such that the observable mass of the universe enclosed within the observed Hubble horizon radius obeys (at any time) the relation $M_{U} \sim R$. Such latter relationship is very similar ( up to a factor of 2 ) ) to the Schwarzschild black-hole horizon-radius relation $r_{s}=2 M$ ( in units of $\hbar=c=G=1$ ). As matter is being created out of the vacuum, the Hubble horizon radius grows accordingly such that $M_{U} / R=c^{2} / G$. ( Note that the Hubble horizon radius is one-half the Schwarzchild horizon radius $\left.(1 / 2)\left(2 G M_{U} / c^{2}\right)=(1 / 2) R_{S}\right)$.

This idea of the Universe as a "primordial" bubble ( like a brane-world ) of Planck size has been also analyzed by [24] from a very different perspective than Born's Dual Phase Space Relativity. These authors have argued that one can have a compatible picture of the expansion of the Universe with the Eddington-Dirac large number coincidences if one invokes a variation of the fundamental constants with the cosmological evolution time as Dirac adovocated long ago. Nottale's proposal [2] for the resolution to the cosmological constant problem is based on taking the Hubble scale $R$ as an upper impassible scale and implementing the Scale Relativity principle so that in order to compare the vacuum energies of the Universe at the Planck scale $\rho\left(L_{P}\right)$ with the vacuum energy measured at the Hubble scale $\rho(R)$ one needs to include the Scale Relativistic correction factors which account for such apparent huge discrepancy : $\rho\left(L_{P}\right) / \rho(R)=\left(R / L_{P}\right)^{2} \sim 10^{122}$. In contrast, the results of this work are based on Born's Dual Phase-Space Relativity principle. In the next sections we will review the dynamical consequences of the Yang's Noncommutative spacetime algebra comprised of two scales, the minimal Planck scale $L_{p}$ ( related to a minimum distance ) and an upper infrared scale $R$ related to a minimum momentum $p=\hbar / R$.

We finalize this subsection by pointing out that the maximal/minimal angular velocity correspond to $c / L_{P}$ and $c / R$ respectively. A maximum angular velocity has important consequences in future Thomas-precession experiments whereas a minimal angular velocity has important consequences in galactic rotation measurements. Maximal acceleration modifications of Schwarszchild geometry have been studied by Lambiase, Pappini, Scarpeta et al [10] over the years. In section 4.4 we wil study the modifications of Newtonian dynamics due to the minimal and maximal length scales using Yang's Noncommutative Spacetime algebra. 


\section{The Extended Relativity in Clifford Spaces}

\subsection{Extending Relativity from Minkowski spacetime to C-space}

We embark into the extended relativity theory in C-spaces by a natural generalization of the notion of a space- time interval in Minkwoski space to C-space:

$$
d X^{2}=d \Omega^{2}+d x_{\mu} d x^{\mu}+d x_{\mu \nu} d x^{\mu \nu}+\ldots
$$

The Clifford valued poly-vector:

$$
X=X^{M} E_{M}=\Omega \mathbf{1}+x^{\mu} \gamma_{\mu}+x^{\mu \nu} \gamma_{\mu} \wedge \gamma_{\nu}+\ldots x^{\mu_{1} \mu_{2} \ldots \mu_{D}} \gamma_{\mu_{1}} \wedge \gamma_{\mu_{2}} \ldots \wedge \gamma_{\mu_{D}}
$$

denotes the position of a polyparticle in a manifold, called Clifford space or $C$-space. The series of terms in (2) terminates at a finite value depending on the dimension $D$. A Clifford algebra $C l(r, q)$ with $r+q=D$ has $2^{D}$ basis elements. For simplicity, the gammas $\gamma^{\mu}$ correspond to a Clifford algebra associated with a flat spacetime :

$$
1 / 2\left\{\gamma^{\mu}, \gamma^{\nu}\right\}=\eta^{\mu \nu}
$$

but in general one could extend this formulation to curved spacetimes with metric $g^{\mu \nu}$.

The connection to strings and p-branes can be seen as follows. In the case of a closed string (a 1-loop) embedded in a target flat spacetime background of $D$-dimensions, one represents the projections of the closed string (1-loop) onto the embedding spacetime coordinate-planes by the variables $x_{\mu \nu}$. These variables represent the respective areas enclosed by the projections of the closed string (1-loop) onto the corresponding embedding spacetime planes. Similary, one can embed a closed membrane (a 2-loop) onto a $D$-dim flat spacetime, where the projections given by the antisymmetric variables $x_{\mu \nu \rho}$ represent the corresponding volumes enclosed by the projections of the 2-loop along the hyperplanes of the flat target spacetimr background.

This procedure can be carried to all closed p-branes ( $\mathrm{p}$-loops ) where the values of $\mathrm{p}$ are $p=0,1,2,3, \ldots . D-2$. The $p=0$ value represents the center of mass and the coordinates $x^{\mu \nu}, x^{\mu \nu \rho} \ldots$ have been coined in the string-brane literature [?] as the holographic areas, volumes, ...projections of the $p$-loops ( closed p-branes ) onto the embedding spacetime coordinate planes/hyperplanes.

Since the D-dimensional Planck scale is given explicitly in terms of the Newton constant : $\Lambda_{D}=\left(G_{N}\right)^{1 /(D-2)}$, in natural units of $\hbar=c=1$, one can see that when $D=\infty$ the value of $\Lambda_{D}$ is then $\Lambda_{\infty}=G^{0}=1$ ( assuming a finite value of $G$ ). Hence in $D=\infty$ the Planck scale has the natural value of unity!. This is important if one wishes to study the convergence property of the series of terms appearing in eq- ( 3.3$)$ below in the extreme case $D=\infty$. For the time being, we shall focus solely on a finite value of $D$ to avoid any serious algebraic convergence problems.

The classification of Clifford algebras $C l(r, q)$ in $D=r+q$ dimensions ( modulo 8 ) for different values of the spacetime signature $r, q$ is discussed, for example, in the book of 
Porteous [27]. All Clifford algebras can be understood in terms of $C L(8)$ and the $C L(k)$ for $k$ less than 8 due to the modulo 8 Periodicity theorem

$$
C L(n)=C L(8) \times C l(n-8)
$$

- $C l(r, q)$ is a matrix algebra for even $n=r+q$ or the sum of two matrix algebras for odd $n=r+q$. Depending on the signature, the matrix algebras may be real, complex, or quaternionic.

If we take the differential $d X$ and compute the scalar product among two polyvectors $<$ $d X^{\dagger} d X>_{\text {scalar }}$ we obtain the C-space extension of the particles proper time in Minkwoski space. The symbol $X^{+}$denotes the reversion operation and involves reversing the order of all the basis $\gamma^{\mu}$ elements in the expansion of $X$. It is the analog of the transpose ( Hermitian ) conjugation. The $\mathrm{C}$-space proper time associated with a polyparticle motion is then :

$$
d \Sigma^{2}=(d \Omega)^{2}+\Lambda^{2 D-2} d x_{\mu} d x^{\mu}+\Lambda^{2 D-4} d x_{\mu \nu} d x^{\mu \nu}+. .
$$

Here we have explicitly introduced the Planck scale $\Lambda$ since a length parameter is needed in order to tie objects of different dimensionality together: 0-loops, 1-loops,..., $p$-loops. Einstein introduced the speed of light as a universal absolute invariant in order to "unite" space with time (to match units) in the Minkwoski space interval:

$$
d s^{2}=c^{2} d t^{2}-d x_{i} d x^{i}
$$

A similar unification is needed here to "unite" objects of different dimensions, such as $x^{\mu}$, $x^{\mu \nu}$, etc... The Planck scale then emerges as another universal invariant in constructing an extended scale relativity theory in C-spaces.

To continue along the same path, we consider the analog of Lorentz transformations in C-spaces which transform a poly-vector $X$ into another poly-vector $X^{\prime}$ given by $X^{\prime}=$ $R X R^{-1}$ with

$$
R=e^{\theta^{A} E_{A}}=\exp \left[\left(\theta \mathbf{1}+\theta^{\mu} \gamma_{\mu}+\theta^{\mu_{1} \mu_{2}} \gamma_{\mu_{1}} \wedge \gamma_{\mu_{2}} \ldots\right)\right]
$$

and

$$
R^{-1}=e^{-\theta^{A} E_{A}}=\exp \left[-\left(\theta \mathbf{1}+\theta^{\nu} \gamma_{\nu}+\theta^{\nu_{1} \nu_{2}} \gamma_{\nu_{1}} \wedge \gamma_{\nu_{2}} \ldots .\right)\right] .
$$

where the theta parameters in $(3.5,3.6)$ are the components of the Clifford-value parameter $\Theta=\theta^{A} E_{A}$ :

$$
\theta ; \theta^{\mu} ; \theta^{\mu \nu} ; \ldots
$$

they are the C-space version of the Lorentz rotations/boosts parameters.

Since a Clifford algebra admits a matrix representation, one can write the norm of a poly-vectors in terms of the trace operation as: $\|X\|^{2}=$ Trace $X^{2}$ Hence under C-space Lorentz transformation the norms of poly-vectors behave like follows:

$$
\text { Trace } X^{\prime 2}=\text { Trace }\left[R X^{2} R^{-1}\right]=\text { Trace }\left[R R^{-1} X^{2}\right]=\text { Trace } X^{2} \text {. }
$$


These norms are invariant under C-space Lorentz transformations due to the cyclic property of the trace operation and $R R^{-1}=1$. There is one word of caution. The condition $\mathbf{R}^{-1}=\tilde{\mathbf{R}}$ that ensures that the inner product $\langle\tilde{X} X\rangle$ is invariant under poly-rotations after using the tilde operation ( the reversal of the gamma basis generators present in the $E_{M}$ polyvectors ) restricts the types of elements allowed in the definition of $\mathbf{R}$. We shall address these issues in section 4 .

\subsection{On the Minimal Planck scale, Superluminal Propagation and C-space Relativity}

Long time ago L.Nottale proposed to view the Planck scale as the absolute minimum invariant (observer independent) scale in Nature in his formulation of scale relativity [?] We can apply this idea to C-spaces by studying the analog of the Minkowski space-time signature $(+,-,-,-)$ by choosing a $\mathrm{C}$-space metric $G^{M N}$ whose signatures relative to the scalar component $\Omega$ of the polyvectors coordinates are :

$$
\begin{gathered}
\|d X\|^{2}=d \Sigma^{2}=(d \Omega)^{2}\left[1-\Lambda^{2 D-2} \frac{\left(d x_{\mu}\right)^{2}}{(d \Omega)^{2}}-\Lambda^{2 D-4} \frac{\left(d x_{\mu \nu}\right)^{2}}{(d \Omega)^{2}}-\Lambda^{2 D-6} \frac{\left(d x_{\mu \nu \rho}\right)^{2}}{(d \Omega)^{2}}-. .\right] \\
\|d X\|^{2}=d \Sigma^{2}=(d \Omega)^{2}\left[1-\left(\frac{\Lambda}{\lambda_{1}}\right)^{2 D-2}-\left(\frac{\Lambda}{\lambda_{2}}\right)^{2 D-4}-\left(\frac{\Lambda}{\lambda_{3}}\right)^{2 D-6}-\ldots\right] .
\end{gathered}
$$

where the sequence of variable scales ( parameters ) $\lambda_{1}, \lambda_{2}, \lambda_{3}, \ldots$ just reflect the magnitudes of the generalized holographic velocities as follows:

$$
\begin{aligned}
& \frac{\left(d x_{\mu}\right)^{2}}{(d \Omega)^{2}} \equiv\left(V_{1}\right)^{2}=\left(\frac{1}{\lambda_{1}}\right)^{2 D-2} . \\
& \frac{\left(d x_{\mu \nu}\right)^{2}}{(d \Omega)^{2}} \equiv\left(V_{2}\right)^{2}=\left(\frac{1}{\lambda_{2}}\right)^{2 D-4} . \\
& \frac{\left(d x_{\mu \nu \rho}\right)^{2}}{(d \Omega)^{2}} \equiv\left(V_{3}\right)^{2}=\left(\frac{1}{\lambda_{3}}\right)^{2 D-6} .
\end{aligned}
$$

etc.... By a simple use of the chain-rule one can relate the velocities defined w.r.t the scalar $\Omega$ variable with those velocities defined with respect to the coordinate-clock variable $t=x^{o}$. For example, $V^{M}=d X^{M} / d \Omega=\left(d X^{M} / d t\right) /(d \Omega / d t)$. It is clear now that if $\|d X\|^{2} \geq 0$ in (3.9) then the sequence of variable lengths $\lambda_{n}$ in (3.10) cannot be smaller than the Planck scale $\Lambda$. This is analogous to a situation with the Minkoswki interval:

$$
d s^{2}=c^{2} d t^{2}\left[1-\frac{v^{2}}{c^{2}}\right]
$$

$d s^{2} \geq 0$ if, and only if, the velocity $v$ does not exceed the speed of light. If any of the $\lambda_{n}$ were smaller than the Planck scale the C-space interval ( 3.9 ) will become tachyonic-like $d \Sigma^{2}<0$. Photons in C-space are tensionless branes/loops. 
This upper holographic-velocity bound does not necessarily translate into a lower bound on the actual values of lengths, areas, volumes.... without the introduction of quantum mechanical considerations [1] . One possibility is that the upper limiting speed of light and the upper bound of the momentum $m_{p} c$ of a Planck-mass elementary particle (the so-called Planckton in the literature) generalizes now to an upper-bound in the $p$-loop holographic velocities and the $p$-loop holographic momenta associated with elementary closed $p$-branes whose tensions are given by powers of the Planck mass. And the latter upper bounds on the holographic $p$-loop momenta implies a lower-bound on the holographic areas, volumes,..., resulting from the string/brane uncertainty relations that we shall derive in the next section.

Thus, Quantum Mechanics is required to implement the postulated principle of minimal lengths, areas, volumes...and which cannot be derived from the classical geometry alone. The emergence of the minimal Planck areas occurs also in the Loop Quantum Gravity program where the expecation values of the Area operator are given by multiples of Planck area. This area-quantization in Planck units can be derived based on the isomorphism of Yang's Noncommutative space-time algebra [29] and the holographic area coordinates algebra in C-space.

To finalize we will discuss briefly the possibility of superluminal propagation [1]. It is known that tachyons can induce a breakdown of causality. The simplest way to see why causality is violated when tachyons are used to exchange signals is by writing the temporal displacements $\delta t=t^{B}-t^{A}$ between two events (in Minkowski space-time) in two different frames of reference:

$$
\begin{gathered}
(\delta t)^{\prime}=(\delta t) \cosh (\xi)+\frac{\delta x}{c} \sinh (\xi)=(\delta t)\left[\cosh (\xi)+\left(\frac{1}{c} \frac{\delta x}{\delta t}\right) \sinh (\xi)\right]= \\
(\delta t)\left[\cosh (\xi)+\left(\beta_{\text {tachyon }}\right) \sinh (\xi)\right] .
\end{gathered}
$$

the boost parameter $\xi$ is defined in terms of the velocity as $\beta_{\text {frame }}=v_{\text {frame }} / c=\tanh (\xi)$, where $v_{\text {frame }}$ is is the relative velocity (in the $x$-direction ) of the two reference frames and can be written in terms of the Lorentz-boost rapidity parameter $\xi$ by using hyperbolic functions. The Lorentz dilation factor is $\cosh (\xi)=\left(1-\beta_{\text {frame }}^{2}\right)^{-1 / 2}$; whereas $\beta_{\text {tachyon }}=$ $v_{\text {tachyon }} / c$ is the beta parameter associated with the tachyon velocity $\delta x / \delta t$. By emitting a tachyon along the negative $x$-direction one has $\beta_{\text {tachyon }}<0$ and such that its velocity exceeds the speed of light $\left|\beta_{\text {tachyon }}\right|>1$ A reversal in the sign of $(\delta t)^{\prime}<0$ in the above boost transformations occurs when the tachyon velocity $\left|\beta_{\text {tachyon }}\right|>1$ and the relative velocity of the reference frames $\left|\beta_{\text {frame }}\right|<1$ obey the inequality condition :

$$
(\delta t)^{\prime}=(\delta t)\left[\cosh (\xi)-\left|\beta_{\text {tachyon }}\right| \sinh (\xi)\right]<0 \Rightarrow 1<\frac{1}{\tanh (\xi)}=\frac{1}{\beta_{\text {frame }}}<\left|\beta_{\text {tachyon }}\right| .
$$

thereby resulting in a causality violation in the primed reference frame since the effect ( event $B$ ) occurs before the cause ( event $A$ ) in the primed reference frame.

In the theory considered here, there are no tachyons in $C$-space, because physical signals in $C$-space are constrained to live inside the $C$-space-light cone [1] . However, certain worldlines in $C$-space, when projected onto the Minkowski subspace $M_{4}$, can appear as 
worldlines of ordinary tachyons outside the lightcone in $M_{4}$. In $C$-space the dynamics refers to a larger space. Minkowski space is just a subspace of $C$-space. "Wordlines" now live in $C$-space that can be projected onto the Minkwoski subspace $M_{4}$. Furthermore, one is enlarging the ordinary Lorentz group to a larger group of C-space Lorentz transformations which involve poly-rotations and generalizations of boosts transformations. In particular, the C-space generalization of the ordinary boost transformations associated with the boost rapidity parameter $\xi$ such that $\tanh (\xi)=\beta_{\text {frame }}$ will involve now the family of C-space boost rapidity parameters $\theta^{t 1}, \theta^{t 12}, \theta^{t 123}, \ldots . \theta^{t 123 \ldots}, \ldots$ since boosts are just ( poly ) rotations along directions involving the time coordinate. Thus, one is replacing the ordinary boost transformations in Minkowski spacetime for the more general C-space boost transformations as we go from one frame of reference to another frame of reference.

Due to the linkage among the C-space coordinates (poly-dimensional covariance) when we envision an ordinary boost along the $x^{1}$ - direction, we must not forget that it is also interconnected to the area-boosts in the $x^{12}$-direction as well, and, which in turn, is also linked to the $x^{2}$ direction. Because the latter direction is transverse to the original tachyonic $x^{1}$-motion, the latter $x^{2}$-boosts won't affect things and we may concentrate on the area-boosts along the $x^{12}$ direction involving the $\theta^{t 12}$ parameter that will appear in the C-space boosts and which contribute to a crucial extra term in the transformations such that no sign-change in $\delta t^{\prime}$ will occur.

More precisely, let us set all the values of the theta parameters to zero except the parameters $\theta^{t 1}$ and $\theta^{t 12}$ related to the ordinary boosts in the $x^{1}$ direction and area-boosts in the $x^{12}$ directions of $\mathrm{C}$-space. This requires, for example, that one has at least one spatial-area component, and one temporal coordinate, which implies that the dimensions must be at least $D=2+1=3$. Thus, we have in this case :

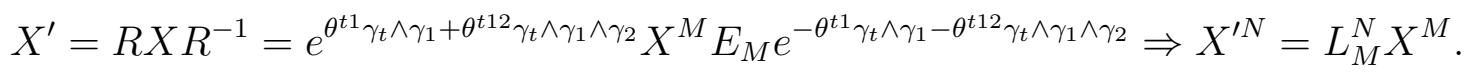

where $L_{M}^{N}$ is given by the scalar part of the Clifford geometric product $L_{M}^{N}=<$ $E^{N} R E_{M} R^{-1}>_{0}$ When one concentrates on the transformations of the time coordinate, we have now that the $\mathrm{C}$-space boosts do not coincide with ordinary boosts in the $x^{1}$ direction :

$$
t^{\prime}=L_{M}^{t} X^{M}=<E^{t} R E_{M} R^{-1}>_{0} X^{M} \neq\left(L_{t}^{t}\right) t+\left(L_{1}^{t}\right) x^{1} .
$$

because of the extra non-vanishing $\theta$ parameter $\theta^{t 12}$.

This is because the rotor $R$ includes the extra generator $\theta^{t 12} \gamma_{t} \wedge \gamma_{1} \wedge \gamma_{2}$ which will bring extra terms into the transformations ; i.e. it will rotate the $E_{[12]}$ bivector- basis , that couples to the holographic coordinates $x^{12}$, into the $E_{t}$ direction which is being contracted with the $E^{t}$ element in the definition of $L_{M}^{t}$. There are extra terms in the C-space boosts because the poly-particle dynamics is taking place in C-space and all coordinates $X^{M}$ which contain the $t, x^{1}, x^{12}$ directions will contribute to the C-space boosts in $D=3$, since one is projecting down the dynamics from $\mathrm{C}$-space onto the $\left(t, x^{1}\right)$ plane when one studies the motion of the tachyon in $M_{4}$. 
Concluding, in the case when one sets all the theta parameters to zero, except the $\theta^{t 1}$ and $\theta^{t 12}$, the $X^{\prime}=R X^{M} E_{M} R^{-1}$ transformations will be :

$$
(\delta t)^{\prime}=L_{M}^{t}\left(\theta^{t 1} ; \theta^{t 12}\right)\left(\delta X^{M}\right) \neq L_{t}^{t}(\delta t)+L_{1}^{t}\left(\delta x^{1}\right) .
$$

due to the presence of the extra term $L_{12}^{t}\left(\delta X^{12}\right)$ in the transformations. In the more general case, when there are more non-vanishing theta parameters, the indices $M$ of the $X^{M}$ coordinates must be restricted to those directions in in C-space which involve the $t, x^{1}, x^{12}, x^{123} \ldots$. directions as required by the $\mathrm{C}$-space poly-particle dynamics. The generalized C-space boosts involve now the ordinary tachyon velocity component of the polyparticle as well as the generalized holographic areas, volumes, hyper-volumes...velocities $V^{M}=\left(\delta X^{M} / \delta t\right)$ associated with the poly-vector components of the Clifford-valued Cspace velocity.

Hence, at the expense of enlarging the ordinary Lorentz boosts to the C-space Lorentz boosts, and the degrees of freedom of a point particle into an extended poly-particle by including the holographic coordinates, in C-space one can still have ordinary pointparticle tachyons without changing the sign of $\delta t$, and without violating causality, due to the presence of the extra terms in the C-space boosts transformations which ensure us that the sign of $\delta t>0$ is maintained as we go from one frame of reference to another one. Naturally, if one were to freeze all the $\theta$ parameters to zero except one $\theta^{t 1}$ one would end up with the standard Lorentz boosts along the $x^{1}$-direction and a violation of causality would occur for tachyons as a result of the sign-change in $\delta t^{\prime}$.

What seems remarkable in this scheme of things is the nature of the signatures and the emergence of two times. One of the latter is the local mode, a clock, represented by $t$ and the other mode is a "global" one represented by the volume of the space-time filling brane. For more details related to this Fock-Stuckelberg-type parameter see [73]. We must emphasize that one must not confuse these global and local time modes with the two modes of time in other branches of science.

Another immediate application of thistheory is that one may consider "strings" and "branes" in C-spaces as a unifying description of all branes of different dimensionality. In fact, a unified action of all p-branes was written in [1] . As we have already indicated, since spinors are left/right ideals elements of a Clifford algebra, a supersymmetry is then naturally incorporated into this approach as well. In particular, one can have world volume and target space supersymmetry simultaneously [44]. We hope that the $C$-space "strings" and "branes" may lead us towards discovering the physical foundations of string and M-theory.

\subsection{The Generalized String/Brane Uncertainty Relations}

Below we will review how the minimal length string uncertainty relations can be obtained fromthe polyparticle dynamics C-spaces [69]. The truly C-space invariant norm of a momentum polyvector is defined (after introducing suitable powers of the Planck scale in the sum in order to match units ) : 


$$
\|P\|^{2}=\pi^{2}+p_{\mu} p^{\mu}+p_{\mu \nu} p^{\mu \nu}+p_{\mu \nu \rho} p^{\mu \nu \rho}+\ldots=M^{2}
$$

A detailed discussion of the physical properties of all the components of the polymomentum $P$ in four dimensions and the emergence of the physical mass $m$ in Minkowski spacetime has been provided in the book by Pavsic [73]. The polymomentum in $D=4$ can be written as :

$$
P=\mu+p^{\mu} \gamma_{\mu}+S^{\mu \nu} \gamma_{\mu} \wedge \gamma_{\nu}+\pi^{\mu} \gamma_{5} \gamma_{\mu}+m \gamma_{5}
$$

where the pseudo-scalar component $m \gamma_{5}$ is the one which contains the physical mass in Minkwoski spacetime. This justifies using the notation $m$ for mass.

The most salient feature of the polyparticle dynamics in C-spaces is that one can start with a constrained action in C-space and arrive, nevertheless, at an unconstrained Stuckelberg action in Minkowski space (a subspace of C-space). It follows that $p_{\mu}$ is a constant of motion $p_{\mu} p^{\mu}=m^{2}$ but $m$ is no longer a fixed constant entering the action but it is now an arbitrary constant of motion. The true constraint in C-space is :

$$
P_{A} P^{A}=\mu^{2}+p_{\mu} p^{\mu}+\pi_{\mu} \pi^{\mu}-m^{2}-2 S^{\mu \nu} S_{\mu \nu}=M^{2} .
$$

This is basically the distinction between the variable $m$ and the fixed constant $M$. The variable $m$ is the conjugate to the Stuckelberg evolution parameter $s$ that allowed Pavsic to propose a natural solution of the problem of time in Quantum Cosmology [73] :

Nottale has given convincing arguments why the notion of dimension is resolution dependent, and at the Planck scale, the minimum attainable distance, the dimension becomes singular, that is blows-up. Setting aside at this moment the potential algebraic convergence problems when $D=\infty$, if we take the dimension at the Planck scale to be infinity, then the norm $P^{2}$ will involve an infinite number of terms. It is precisely this infinite series expansion which will reproduce all the different forms of the Casimir invariant masses appearing in kappa-deformed Poincare algebras As mentioned earlier, when $D=\infty$, the Planck scale appearing in the series expansion of ( 3.12$) \Lambda_{\infty}=G^{1 / 0}=1$.

It was discussed recently why there is an infinity of possible values of the Casimirs invariant $M^{2}$ due to an infinite choice of possible bases. The parameter $\kappa$ is taken to be equal to the inverse of the Planck scale. The classical Poincare algebra is retrieved when $\Lambda=0$. The kappa-deformed Poincare algebra does not act in classical Minkwoski spacetime. It acts in a quantum-deformed spacetime. We conjecture that the natural deformation of Minkowski spacetime is given by C-space.

The way to generate all the different forms of the Casimirs $M^{2}$ is by "projecting down" from the $2^{D}$-dim Clifford algebra to $D$-dim. One simply "slices" the $2^{D}$-dim massshell hyper-surface in C-space by a $D$-dimensional one. This is achieved by imposing the following constraints on the holographic components of the polyvector-momentum. In doing so one is explicitly breaking the poly-dimensional covariance and for this reason one can obtain an infinity of possible choices for the Casimirs $M^{2}$.

To demonstrate this, we impose the following constraints :

$$
p_{\mu \nu} p^{\mu \nu}=a_{2}\left(p_{\mu} p^{\mu}\right)^{2}=a_{2} p^{4} . \quad p_{\mu \nu \rho} p^{\mu \nu \rho}=a_{3}\left(p_{\mu} p^{\mu}\right)^{3}=a_{3} p^{6} . \quad \ldots \ldots
$$


What eqs-(3-13 ) represent geometrically is the slicing of the $2^{D}$-dimensional massshell hypersurface in $\mathrm{C}$-space into $D$-dimensional regions (subspaces) parametrized solely in terms of the ordinary momentum coordinates $p_{\mu}$. This is the reason why decided to choose such constraint ( 3.13 ). There are many different ways to perform the slicing procedure in C-space depending on the choices of the coefficients $a_{n}$. Upon doing so the norm of the poly-momentum becomes:

$$
P^{2}=\sum_{n} a_{n} p^{2 n}=M^{2}\left(1, a_{2}, a_{3}, \ldots, a_{n}, \ldots\right)
$$

Therefore, by a judicious choice of the coefficients $a_{n}$, and by reinserting the suitable powers of the Planck scale, which have to be there in order to combine objects of different dimensions, one can reproduce all the possible Casimirs in the form:

$$
M^{2}=m^{2}[f(\Lambda m / \hbar)]^{2} . \quad m^{2} \equiv p_{\mu} p^{\mu}=p^{2} .
$$

To illustrate the relevance of polyvectors, we will summarize our derivation of the minimal length string uncertainty relations Because of the existence of the extra holographic variables $x^{\mu \nu}, \ldots$ one cannot naively impose $[x, p]=i \hbar$ due to the effects of the other components. The units of $\left[x_{\mu \nu}, p^{\mu \nu}\right]$ are of $\hbar^{2}$ and of higher powers of $\hbar$ for the other commutators. To achieve covariance in $\mathrm{C}$-space which reshuffles objects of different dimensionality, the effective Planck constant in C-space should be given by a sum of powers of $\hbar$.

This is not surprising. Classical C-space contains the Planck scale, which itself depends on $\hbar$. This implies that already at the classical level, C-space contains the seeds of the quantum space. At the next level of quantization, we have an effective $\hbar$ that comprises all the powers of $\hbar$ induced by the commutators involving all the holographic variables. In general one must write down the commutation relations in terms of polyvector- valued quantities. In particular, the Planck constant will now be a Clifford number, a polyvector with multiple components [29].

The simplest way to infer the effects of the holographic coordinates of C-space on the commutation relations is by working with the effective $\hbar$ that appears in the nonlinear de Broglie dispersion relation. The mass-shell condition in C-space, after imposing the constraints among the holographic components, yields an effective mass $M=m f(\Lambda m / \hbar)$. The generalized De Broglie relations are no longer linear:

$$
\begin{gathered}
\left|P_{\text {effective }}\right|=|p| f(\Lambda m / \hbar)=\hbar_{\text {effective }}|k| . \quad \hbar_{\text {effective }}=\hbar f(\Lambda m / \hbar)= \\
\hbar \sum_{n=0}^{n=N} a_{n}(\Lambda m / \hbar)^{2 n} . \quad m^{2}=p^{2}=p_{\mu} p^{\mu}=(\hbar k)^{2} .
\end{gathered}
$$

where the upper limit in the sum $N=D$.

Using the effective $\hbar_{e f f}$, the well known relation based on the Schwartz inequality and the fact that $|z| \geq|\operatorname{Im} z|$ we obtain:

$$
\Delta x^{i} \Delta p^{j} \geq \frac{1}{2}\left\|<\left[x^{i}, p^{j}\right]>\right\| . \quad\left[x^{i}, p^{j}\right]=i \hbar_{e f f} \delta^{i j} .
$$


In Euclidean space-time one has that the norms:

$$
\hbar^{2} k^{2}=\left\|p_{\mu} p^{\mu}\right\|=m^{2}=\left\|\left(p_{0}\right)^{2}+\vec{p}^{2}\right\| \geq\left\|\vec{p}^{2}\right\| . \quad m^{2} \geq\left\|\vec{p}^{2}\right\| .
$$

Choosing a positive sign of the numerical coefficients $a_{n}>0$ in eq-(3.16) one has :

$$
\begin{gathered}
a_{n} m^{2 n} \geq a_{n}\left\|\vec{p}^{2}\right\|^{n}=a_{n}\left[\left(p_{1}\right)^{2}+\left(p_{2}\right)^{2}+\ldots .\left(p_{D-1}\right)^{2}\right]^{n} \Rightarrow \\
a_{n} m^{2 n} \geq a_{n}\left(p_{1}\right)^{2 n} . \quad a_{n} m^{2 n} \geq a_{n}\left(p_{2}\right)^{2 n} . \quad a_{n} m^{2 n} \geq a_{n}\left(p_{3}\right)^{2 n} \ldots \ldots .
\end{gathered}
$$

Therefore, from eq-( 3.19$)$ in conjunction with the inequalities:

$$
<p^{2}>\geq(\Delta p)^{2} . \quad<p^{4}>\geq(\Delta p)^{4} \ldots \ldots
$$

and upon using the series expansion (3-16) of the effective $\hbar_{\text {eff }}$ that appears in the r.h.s of ( $3-17)$, we get for each component (we omit indices for simplicity) :

$$
\Delta x \Delta p \geq \frac{1}{2} \hbar+\frac{a_{1} \Lambda^{2}}{2 \hbar}(\Delta p)^{2}+\ldots \cdots \cdots
$$

This yields the minimal length string uncertainty relations with corrections :

$$
\Delta x \geq \frac{\hbar}{2 \Delta p}+\frac{a_{1} \Lambda^{2}}{2 \hbar} \Delta p+\ldots
$$

By replacing lengths by times and momenta by energy one reproduces the minimal Planck time uncertainty relations.

The Physical interpretation of these uncertainty relations follow from the extended relativity principle. As we boost the string to higher trans-Planckian energies, part of the energy will always be invested into the strings potential energy, increasing its length intobits of Planck scale sizes, so that the original string will decompose into two, three, four....strings of Planck sizes carrying units of Planck momentum; i.e. the notion of $a$ single particle/string loses its meaning beyond that point.

This reminds one of ordinary relativity, where boosting a massive particle to higher energy increasesthe speed whilepart of the energy is also invested into increasing its mass. In this process the speed of light remains the maximum attainable speed (it takes an infinite energyto reach it) and in our scheme the Planck scale is never surpassed. The effects of a minimal length can be clearly seen in Finsler geometries [12] having both a maximum four acceleration $c^{2} / \Lambda$ (maximum tidal forces) and a maximum speed . The Riemannian limit is reached when the maximum four acceleration goes to infinity; i.e. The Finsler geometry "collapses" to a Riemannian one. 


\section{The Extended Relativity in Born-Clifford Phase Spaces}

\subsection{The Clifford-Phase Space with a Lower and Upper Length scales}

Our task now is to construct a Relativity theory that implements simultaneously the minimal and maximal scale Relativity principle. One should expect a relationship with Yang's Noncommutative Spacetime algebra, with an upper and a lower scale associated with the embedding of $A d S_{5}$ into a $6 D$ pseudo-Euclidean space. The upper scale $R$ is related to the throat-size of $A d S_{5}$, an infrared regulator. The minimal scale is set equal to the Planck length $L_{P}$.

The interval in Clifford-Phase-Spaces is defined :

$$
\begin{gathered}
d \Sigma^{2}=<d X^{\dagger} d X>+\frac{1}{\mathcal{F}^{2}}<d P^{\dagger} d P>= \\
\left(\frac{d \Omega}{L_{P}^{D-1}}\right)^{2}+d x_{\mu} d x^{\mu}+\frac{d x_{\mu \nu} d x^{\mu \nu}}{L_{P}^{2}}+\frac{d x_{\mu \nu \rho} d x^{\mu \nu \rho}}{L_{P}^{4}}+\ldots \\
\frac{1}{\mathcal{F}^{2}}\left[\frac{(d \tilde{\Omega})^{2}}{(\hbar / R)^{2 D-2}}+d p_{\mu} d p^{\mu}+\frac{d p_{\mu \nu} d p^{\mu \nu}}{(\hbar / R)^{2}}+\frac{d p_{\mu \nu \rho} d p^{\mu \nu \rho}}{(\hbar / R)^{4}}+\ldots \ldots\right]
\end{gathered}
$$

where the maximal force :

$$
\mathcal{F}=\frac{m_{P} c^{2}}{L_{P}}=\frac{M_{U} c^{2}}{R}=\frac{c^{4}}{G}
$$

The infrared scale $R \rightarrow \infty$ limit is set in conjunction with the vanishing limit of the holographic momenta variables (to avoid singularities)

$$
p^{\mu \nu} \rightarrow 0, p^{\mu \nu \rho} \rightarrow 0, \ldots
$$

constraining the interval in the Born-Clifford Phase Space :

$$
\begin{gathered}
d \Sigma^{2} \Rightarrow \frac{(d \Omega)^{2}}{L_{P}^{2 D-2}}+d x_{\mu_{1}} d x^{\mu_{1}}+\frac{d x_{\mu_{1} \mu_{2}} d x^{\mu_{1} \mu_{2}}}{L_{P}^{2}}+\frac{d x_{\mu_{1} \mu_{2} \mu_{3}} d x^{\mu_{1} \mu_{2} \mu_{3}}}{L_{P}^{4}}+\ldots \\
\frac{d x_{\mu_{1} \mu_{2} \mu_{3} \ldots \mu_{D-1}} d x^{\mu_{1} \mu_{2} \mu_{3} \ldots \mu_{D-1}}}{L_{P}^{2 D-2}}+\frac{1}{\mathcal{F}^{2}} d p_{\mu} d p^{\mu} .
\end{gathered}
$$

In the classical limit $\hbar \rightarrow 0$ the Planck scale $L_{P}=\sqrt{\left(\hbar G / c^{3}\right)} \rightarrow 0$. Thus, the ultraviolet limit $L_{P} \rightarrow 0$ is accompanied with the vanishing limit of the holographic coordinate variables

$$
x^{\mu \nu} \rightarrow 0, x^{\mu \nu \rho} \rightarrow 0, \ldots
$$

and the interval reduces further to Born Phase Space interval :

$$
d \Sigma^{2} \Rightarrow d x_{\mu} d x^{\mu}+\frac{1}{\mathcal{F}^{2}} d p_{\mu} d p^{\mu}=
$$




$$
\begin{gathered}
d \tau^{2}\left[1+\frac{\left(d p_{\mu} / d \tau\right)\left(d p^{\mu} / d \tau\right)}{\mathcal{F}^{2}}\right]= \\
d \tau^{2}\left[1+\frac{1}{\mathcal{F}^{2}} m^{2} \frac{d^{2} x_{\mu}}{d \tau^{2}} \frac{d^{2} x^{\mu}}{d \tau^{2}}\right]=d \tau^{2}\left[1-\frac{m^{2} g^{2}(\tau)}{\mathcal{F}^{2}}\right] .
\end{gathered}
$$

When $\mathcal{F} \rightarrow \infty$ the Phase Space interval reduces further to the Minkowski spacetime interval.

As stated above, when $\hbar \rightarrow 0$ one has that the Planck scale $L_{P} \rightarrow 0$ and also one should notice that :

$$
m_{P}=\frac{\hbar}{c L_{P}}=\frac{\hbar}{c \sqrt{\left(\hbar G / c^{3}\right)}} \rightarrow 0 . \quad a_{\max }=\frac{c^{2}}{L_{P}}=\sqrt{\left(c^{7} / \hbar G\right)} \rightarrow \infty . \quad m_{P} a_{\max }=\frac{c^{4}}{G}=\mathcal{F} .
$$

Despite that the maximal acceleration is infinite this does not necessarily imply that the maximal force is also divergent due to the fact that the $m_{P} \rightarrow 0$.

Hence there are two regimes that interpolate from the Born-Clifford Phase Space interval to the standard point-particle Born Phase Space interval (related to the existence of a maximal force). In the infinite distance (infrared ) limit $R \rightarrow \infty$ the minimum momentum collapses to zero and in order to avoid singularities we must set all the holographic momenta to zero that leads to the decoupling of the holographic momenta variables. In the classical limit $\hbar \rightarrow 0$ the Planck scale collapses to zero and in order to avoid singularities we must set all the holographic coordinate variables to zero so one ends up solely with the Born's Dual Phase Space Relativity interval associated with a point particle subjected to an upper bound on the force $\mathcal{F}=c^{4} / G$ and with an upper bound on the speed given by $c$.

\subsection{A Clifford realization of Velocity and Acceleration Boosts in Phase Spaces}

Introducing complex coordinates :

$$
z^{\mu}=x^{\mu}+i \frac{p^{\mu}}{\mathcal{F}} . \quad z^{\mu \nu}=\frac{x^{\mu \nu}}{L_{P}}+i \frac{p^{\mu \nu}}{\mathcal{F}(\hbar / R)} \cdots
$$

along with their complex conjugates will be very useful as we shall see next. Upon defining the maximal force $\mathcal{F}=b$ the effective boost parameter $\xi$ can be written in terms of the the velocity boosts $\xi_{v}\left(\tanh \left(\xi_{v} / c\right)=v / c\right)$ and acceleration boosts parameter $\xi_{a}($ $\left.\tanh \left(\xi_{a} / b\right)=m a / m_{P} a_{\max }\right)$ as :

$$
\xi=\frac{\xi_{v}}{c}+i \frac{\xi_{a}}{\mathcal{F}} . \quad\|\xi\|=\sqrt{\xi \bar{\xi}}=\sqrt{\left(\xi_{v} / c\right)^{2}+\left(\xi_{a} / \mathcal{F}\right)^{2}} . \quad 4-7
$$

From the transformations of the $(X, T, E, P)$ variables given in eqs- $($ ) one finds:

$$
z_{1}^{\prime}=z_{1} \cosh (\|\xi\|)+z_{0}\left(\xi_{v} / c+i \xi_{a} / b\right) \frac{\sinh (\|\xi\|)}{\|\xi\|} \quad 4-8
$$




$$
\begin{array}{lc}
z_{0}^{\prime}=z_{0} \cosh (\|\xi\|)+z_{1}\left(\xi_{v} / c-i \xi_{a} / b\right) \frac{\sinh (\|\xi\|)}{\|\xi\|} . & 4-9 \\
\bar{z}_{1}^{\prime}=\bar{z}_{1} \cosh (\|\xi\|)+\bar{z}_{0}\left(\xi_{v} / c-i \xi_{a} / b\right) \frac{\sinh (\|\xi\|)}{\|\xi\|} & 4-10 \\
\bar{z}_{0}^{\prime}=\bar{z}_{0} \cosh (\|\xi\|)+\bar{z}_{1}\left(\xi_{v} / c+i \xi_{a} / b\right) \frac{\sinh (\|\xi\|)}{\|\xi\|} . & 4-11
\end{array}
$$

These transformations leave invariant the Phase Space interval rewritten in complex coordinates:

$$
\begin{array}{lr}
z_{0} \bar{z}_{0}-z_{1} \bar{z}_{1}=z_{0}^{\prime} \bar{z}_{0}^{\prime}-z_{1}^{\prime} \bar{z}_{1}^{\prime}=\text { invariant. } & 4-12 \\
(d \Sigma)^{2}=d \omega d \bar{\omega}+d z_{\mu} d \bar{z}^{\mu}+d z_{\mu \nu} d \bar{z}^{\mu \nu}+\ldots & 4-13
\end{array}
$$

The natural symmetry group action in the Clifford-Phase Space associated with $8 D$ is the Clifford group $C l(8)$ with $2^{8}=256$ elements. This $C l(8)$ group contains naturally the Spin(8) group with 28 elements associated with the bivectors basis generators of the Clifford algebra. Namely with ordinary rotations and boosts in $8 D$. The $\operatorname{Spin}(8)$ breaks up into an $U(4)$ piece associated with the MacDowell-Mansouri-ChamseddineWest formulation of gravity and the $S U(3) \otimes S U(2) \otimes U(1)$ associated with the Standard Model . Since $U(1,3)$ is the symmetry group studied by Low comprised of velocity and acceleration boosts, rotations,.. in the $8 D$ Phase Space which leave invariant the ordinary point-particle Phase Space interval, it is fairly clear that the Clifford group $C l(8)$ ( which contains the $\operatorname{Spin}(8)$ group ) contains the required $U(4)$ piece and is going to be large enough to implement the generalized velocity and acceleration boosts of the holographic Clifford-valued coordinates and momenta in the Clifford-Phase-Space interval .

The signature of the $8 D$ Phase space $x^{0}, p^{0}, x^{1}, p^{1}, x^{2}, p^{2}, \ldots$ is chosen to be $(+,+,-,-,-,-\ldots-)$; i.e. there are two timelike and six spacelike directions. We choose to split the $8 D$ Clifford algebra generators into pairs of $\gamma^{\prime}$ 's and $\beta$ 's as follows :

$$
\Gamma_{0}=\gamma_{0} . \quad \Gamma_{1}=\gamma_{1}, \quad \Gamma_{2}=\gamma_{2} . \quad \Gamma_{3}=\gamma_{3} . \quad \Gamma_{4}=\beta_{0}, \quad \Gamma_{5}=\beta_{1}, \quad \Gamma_{6}=\beta_{2}, \quad \Gamma_{7}=\beta_{3} \quad 4-14
$$

obeying the relations :

$$
\left\{\gamma_{\mu}, \gamma_{\nu}\right\}=2 \eta^{\mu \nu} . \quad\left\{\beta_{\mu}, \beta_{\nu}\right\}=2 \eta^{\mu \nu} . \quad\left\{\gamma_{\mu}, \beta_{\nu}\right\}=0 . \quad\left\{\beta_{\mu}, \gamma_{\nu}\right\}=0 \quad 4-15
$$

Thus the squares of the gamma basis are :

$$
\gamma_{0}^{2}=\beta_{0}^{2}=1 . \quad \gamma_{1}^{2}=\beta_{1}^{2}=-1 . \quad \gamma_{2}^{2}=\beta_{2}^{2}=-1 . \quad \gamma_{3}^{2}=\beta_{3}^{2}=-1 . \quad \ldots . \quad 4-16
$$

we also have the important relations

$$
\begin{gathered}
\left(\gamma_{0} \beta_{0}\right)\left(\gamma_{0} \beta_{0}\right)=-\gamma_{0}^{2} \beta_{0}^{2}=-1 . \quad\left(\gamma_{1} \beta_{1}\right)\left(\gamma_{1} \beta_{1}\right)=-\gamma_{1}^{2} \beta_{1}^{2}=-1 \ldots \\
\gamma_{5}=\gamma_{0} \gamma_{1} \gamma_{2} \gamma_{3} . \quad \gamma_{5}^{2}=-1 .
\end{gathered}
$$




$$
\beta_{5}=\beta_{0} \beta_{1} \beta_{2} \beta_{3} . \quad \beta_{5}^{2}=-1 .
$$

Notice that due to the $(2,6)$ signature one has $\left(\gamma_{5} \beta_{5}\right)^{2}=1$. The Clifford algebras $C l(2,6, R), C l(6,2, R)$ can be realized in terms of the matrix algebra $\mathcal{M}(8, \mathbf{H})$ given by $8 \times 8$ Quaternionic entries.

To illustrate the usefulness of Clifford algebras it is convenient to derive the ordinary velocity boosts transformations along the $x_{1}$ direction using Clifford algebras. Since the directions $x_{2}$ and $x_{3}$ are unaffected one may concentrate solely on the real $2 D$ Clifford algebra $C l(1,1 ; R)$ that can be realized in terms of the matrix algebra $\mathcal{M}(2, R)$ comprised of $2 \times 2$ real matrices.

A Clifford algebra in $2 D$ has four generators $1, \gamma_{0}, \gamma_{1}$ and the bivector $\gamma_{0} \gamma_{1}$. Because $\gamma_{0}^{2}=1$ and $\gamma_{1}^{2}=-1$, the $\gamma_{0}$ element can be realized as a $2 \times 2$ traceless diagonal matrix with entries 1, -1 along the diagonal. $\gamma_{0}$ is Hermitian $\gamma_{0}^{\dagger}=\gamma_{0}$ whereas the anti-Hermitian traceless $\gamma_{1}$ can be realized as $i$ times an off-diagonal $2 \times 2$ matrix whose entries are 1,1 and such as $\gamma_{1}^{2}=-1$. The bivector $\gamma_{0} \gamma_{1}$ is Hermitian $\left(\gamma_{0} \gamma_{1}\right)^{\dagger}=\gamma_{1}^{\dagger} \gamma_{0}^{\dagger}=-\gamma_{1} \gamma_{0}=\gamma_{0} \gamma_{1}$ since the $\gamma$ 's anti-commute. Thus the traceless Hermitian $2 \times 2$ matrix $\gamma_{0} \gamma_{1}$ is given by $i$ times an anti-symmetric ( off diagonal ) matrix whose entries are 1, -1 respectively.

The rotor corresponding to an ordinary velocity boosts transformations in coordinate space along the $x_{1}$ directions :

$$
\mathbf{X}^{\prime}=x_{0}^{\prime} \gamma^{0}+x_{1}^{\prime} \gamma^{1}=\mathbf{R}\left(x_{0} \gamma^{0}+x_{1} \gamma^{1}\right) \mathbf{R}^{-1}
$$

is defined as :

$$
\mathbf{R}=\exp \left[\frac{1}{2} \frac{\xi_{v}}{c} \gamma_{0} \gamma_{1}\right]=\cosh \left(\frac{1}{2}\|\xi\|\right)+\gamma_{0} \gamma_{1} \sinh \left(\frac{1}{2}\|\xi\|\right) . \quad 4-20
$$

where $\|\xi\|=\frac{\xi_{v}}{c}$ when $\xi_{a}=0$.

$$
\mathbf{R}^{-1}=\exp \left[-\frac{1}{2} \frac{\xi_{v}}{c} \gamma_{0} \gamma_{1}\right]=\cosh \left(\frac{1}{2}\|\xi\|\right)-\gamma_{0} \gamma_{1} \sinh \left(\frac{1}{2}\|\xi\|\right) . \quad 4-21
$$

After performing a Taylor series expansion of the exponential we arrive at the expressions for $\mathbf{R}$ and $\mathbf{R}^{-1}$ explicitly in terms of hyperbolic functions due to the non-compact nature of the Lorentz group. The Taylor series expansion is based on the following conditions :

$$
\left\{\gamma_{0}, \gamma_{1}\right\}=0 \Rightarrow\left(\gamma_{0} \gamma_{1}\right)^{2}=\gamma_{0} \gamma_{1} \gamma_{0} \gamma_{1}=-\gamma_{0}^{2} \gamma_{1}^{2}=-(1)(-1)=+1 \quad 4-22
$$

so that the square :

$$
\frac{1}{4}\left(\frac{\xi_{v}}{c} \gamma_{0} \gamma_{1}\right)^{2}=\frac{1}{4}\left(\frac{\xi_{v}^{2}}{c^{2}}\right)^{2}=\|\xi / 2\|^{2} . \quad\left(\gamma_{0} \gamma_{1}\right)^{2}=1
$$

The cube becomes after multiplying and dividing by $\frac{1}{2}\|\xi\|$ :

$$
\|\xi / 2\|^{3} \frac{1}{(\|\xi\| / 2)} \frac{1}{2}\left[\frac{\xi_{v}}{c} \gamma_{0} \gamma_{1}\right]
$$


In this way the even powers will combine to give the $\cosh \left(\frac{1}{2}|| \xi \|\right)$ term and the odd powers will combine to give the term :

$$
\frac{1}{\|\xi\|} \sinh \left(\frac{1}{2}\|\xi\|\right)\left[\frac{\xi_{v}}{c} \gamma_{0} \gamma_{1}\right]
$$

The identities :

$$
\cosh (\|\xi\|)=\cosh ^{2}\left(\frac{1}{2}\|\xi\|\right)+\sinh ^{2}\left(\frac{1}{2}\|\xi\|\right) . \quad \sinh (\|\xi\|)=2 \sinh \left(\frac{1}{2}\|\xi\|\right) \cosh \left(\frac{1}{2}\|\xi\|\right)
$$

allow us to evaluate:

$$
\begin{gathered}
\mathbf{X}^{\prime}=x_{0}^{\prime} \gamma^{0}+x_{1}^{\prime} \gamma^{1}=\mathbf{R X R}^{-1}= \\
{\left[\cosh \left(\frac{1}{2}\|\xi\|\right)+\gamma_{0} \gamma_{1} \sinh \left(\frac{1}{2}\|\xi\|\right)\right]\left[x_{0} \gamma^{0}+x_{1} \gamma^{1}\right]\left[\cosh \left(\frac{1}{2}\|\xi\|\right)-\gamma_{0} \gamma_{1} \sinh \left(\frac{1}{2}\|\xi\|\right)\right]=} \\
{\left[x_{0} \cosh (\|\xi\|)-x_{1} \sinh (\|\xi\|)\right] \gamma^{0}+\left[x_{1} \cosh (\|\xi\|)-x_{0} \sinh (\|\xi\|) \gamma^{1}=\right.} \\
x_{0}^{\prime} \gamma^{0}+x_{1}^{\prime} \gamma^{1}
\end{gathered}
$$

from which we can immediately read-off the transformations

$$
x_{0}^{\prime}=x_{0} \cosh (\|\xi\|)-x_{1} \sinh (\|\xi\|) . \quad x_{1}^{\prime}=x_{1} \cosh (\|\xi\|)-x_{0} \sinh (\|\xi\|) . \quad 4-30
$$

that leave invariant the indefinite quadratic form $x_{0}^{2}-x_{1}^{2}$ defined as :

$$
<\mathbf{X}^{\prime} \mathbf{X}^{\prime}>_{\text {scalar }}=\left(x_{0}^{\prime}\right)^{2}-\left(x_{1}^{\prime}\right)^{2}=<\mathbf{X X}>_{\text {scalar }}=\left(x_{0}\right)^{2}-\left(x_{1}\right)^{2} \quad 4-31
$$

since the reversal of the vector $\mathbf{X}$ is itself and $\gamma_{0}^{2}=1, \gamma_{1}^{2}=-1$ and $\left\{\gamma_{0}, \gamma_{1}\right\}=0$. which explains why there are no mixed terms $x_{0} x_{1}$ in the indefinite quadratic form.

The indefinite quadratic form can also be recast as a minus the determinant of the $2 \times 2$ matrix:

$$
-\operatorname{det}\left(\mathbf{X}^{\prime}\right)=\left(x_{0}^{\prime}\right)^{2}-\left(x_{1}^{\prime}\right)^{2}=-\operatorname{det}(\mathbf{R}) \operatorname{det}(\mathbf{X}) \operatorname{det}\left(\mathbf{R}^{-1}\right)=\left(x_{0}\right)^{2}-\left(x_{1}\right)^{2} \quad 4-32
$$

. since $\operatorname{det}\left(\mathbf{R}^{-1}\right) \operatorname{det}(\mathbf{R})=1$.

\subsection{The Clifford Cl (2, 6, R ) Symmetry Group}

The Phase-Space transformations require much more work due to the mixing of coordinates and momenta in order to implements Born's Dual Relativity Principle in PhaseSpaces that we postulated as the underlying origins of $T$ duality symmetry in string/M theory. Since we will be studying velocity and acceleration boosts ( force boosts) along the $x_{1}$ and $p_{1}$ directions, it is convenient to write only the relevant variables affected by 
these transformations. Thus, we shall forget about the $x_{2}, p_{2}, x_{3}, p_{3}$ coordinates for convenience purposes and focus at the moment on the $4 D$ phase Space associated with the $x_{0}, p_{0}, x_{1}, p_{1}$ variables .

Defining the $4 D$ Phase space vector :

$$
\mathbf{Z}=x^{0} \gamma_{0}+p^{0} \beta_{0}+x^{1} \gamma_{1}+p^{1} \beta_{1} . \quad \mathbf{Z}^{\prime}=\mathbf{R Z R}^{-1}
$$

where for convenience purposes we have written $p$ instead of $p / \mathcal{F}$. The rotor is now given by :

$$
\mathbf{R}=\exp \left[\frac{1}{2}\left(\frac{\xi_{v}}{c} \Upsilon_{v}+\frac{\xi_{a}}{b} \Upsilon_{a}\right)\right]
$$

where $\Upsilon_{v}$ and $\Upsilon_{a}$ are the sought-after velocity and acceleration boosts in the $4 D$ PhaseSpace. These generators are suitable linear combinations of the bi-vectors basis elements associated with the Clifford algebra $C l(2,2)$ which is isomorphic to a matrix algebra $\mathcal{M}(4, \mathbf{R})$ comprised of $4 \times 4$ real matrices. In this subsection we shall follow all the steps necessary to determine the form of $\Upsilon_{v}$ and $\Upsilon_{a}$ that reproduce the $U(1,1)$ transformations in complex coordinates form. It is a non trivial exercise.

Firstly, in order to perform the Taylor series expansion of the exponentials, one requires the following 3 orthonormality conditions to begin with :

$$
\left\{\Upsilon_{v}, \Upsilon_{a}\right\}=0 . \quad \Upsilon_{v}^{2}=1 . \quad \Upsilon_{a}^{2}=1
$$

so that the square :

$$
\left(\frac{\xi_{v}}{c} \Upsilon_{v}+\frac{\xi_{a}}{b} \Upsilon_{a}\right)^{2}=\frac{\xi_{v}^{2}}{c^{2}}+\frac{\xi_{a}^{2}}{b^{2}}=\|\xi\|^{2}
$$

Hence the mixed terms cancel out due to the "orthogonality" condition $\left\{\Upsilon_{v}, \Upsilon_{a}\right\}=0$. Thus, the square of the exponents in the definition of $\mathbf{R}$ becomes :

$$
\left[\frac{1}{2}\left(\frac{\xi_{v}}{c} \Upsilon_{v}+\frac{\xi_{a}}{b} \Upsilon_{a}\right)\right]^{2}=\frac{1}{4}\left(\frac{\xi_{v}^{2}}{c^{2}}+\frac{\xi_{a}^{2}}{b^{2}}\right)=\left\|\frac{\xi}{2}\right\|^{2}
$$

The cube becomes after multiplying and dividing by $\| \xi / 2||$ :

$$
\left\|\frac{\xi}{2}\right\|^{3} \frac{1}{(\|\xi\| / 2)} \frac{1}{2}\left[\frac{\xi_{v}}{c} \Upsilon_{v}+\frac{\xi_{a}}{b} \Upsilon_{a}\right]
$$

In this way the even powers will combine to give the $\cosh \left(\frac{1}{2}|| \xi||\right)$ term and the odd powers will combine to give the term :

$$
\frac{1}{\|\xi\|} \sinh \left(\frac{1}{2}\|\xi\|\right)\left[\frac{\xi_{v}}{c} \Upsilon_{v}+\frac{\xi_{a}}{b} \Upsilon_{a}\right]
$$

the rotor becomes :

$$
\mathbf{R}=\cosh \left(\frac{1}{2}\|\xi\|\right)+\left[\frac{\xi_{v}}{c} \Upsilon_{v}+\frac{\xi_{a}}{b} \Upsilon_{a}\right] \frac{\sinh \left(\frac{1}{2}\|\xi\|\right)}{\|\xi\|}
$$

The inverse is : 


$$
\mathbf{R}^{-1}=\mathbf{R}^{\dagger}=\cosh \left(\frac{1}{2}\|\xi\|\right)-\left[\frac{\xi_{v}}{c} \Upsilon_{v}+\frac{\xi_{a}}{b} \Upsilon_{a}\right] \frac{\sinh \left(\frac{1}{2}\|\xi\|\right)}{\|\xi\|}
$$

The following 4 relations

$$
\begin{array}{rlrl}
{\left[\frac{\xi_{v}}{c} \Upsilon_{v}+\frac{\xi_{a}}{b} \Upsilon_{a}\right] \gamma_{0}\left[\frac{\xi_{v}}{c} \Upsilon_{v}+\frac{\xi_{a}}{b} \Upsilon_{a}\right]} & =-\gamma_{0}\|\xi\|^{2} . & 4-42 a \\
{\left[\frac{\xi_{v}}{c} \Upsilon_{v}+\frac{\xi_{a}}{b} \Upsilon_{a}\right] \gamma_{1}\left[\frac{\xi_{v}}{c} \Upsilon_{v}+\frac{\xi_{a}}{b} \Upsilon_{a}\right]} & =-\gamma_{1}\|\xi\|^{2} . & 4-42 b \\
{\left[\frac{\xi_{v}}{c} \Upsilon_{v}+\frac{\xi_{a}}{b} \Upsilon_{a}\right] \beta_{0}\left[\frac{\xi_{v}}{c} \Upsilon_{v}+\frac{\xi_{a}}{b} \Upsilon_{a}\right]=-\beta_{0}\|\xi\|^{2} .} & 4-42 c \\
{\left[\frac{\xi_{v}}{c} \Upsilon_{v}+\frac{\xi_{a}}{b} \Upsilon_{a}\right] \beta_{1}\left[\frac{\xi_{v}}{c} \Upsilon_{v}+\frac{\xi_{a}}{b} \Upsilon_{a}\right]=-\beta_{1}\|\xi\|^{2} .} & 4-42 d
\end{array}
$$

are direct consequences of the 8 flip conditions below that need to be satisfied bringing the total number of independent conditions to $3+8=11$ comprised of 3 orthonormality and 8 flip conditions.

$3+8=11=$ mebrane

Therefore, we must find 11 independent coefficients in the defining relations for the generators $\Upsilon_{v}$ and $\Upsilon_{a}$.

The 8 flip conditions are :

$$
\begin{array}{ll}
{\left[\frac{\xi_{v}}{c} \Upsilon_{v}+\frac{\xi_{a}}{b} \Upsilon_{a}\right] \gamma_{0}=\left[\frac{\xi_{v}}{c}+i \frac{\xi_{a}}{b}\right] \gamma_{1}} & 4-43 a \\
{\left[\frac{\xi_{v}}{c} \Upsilon_{v}+\frac{\xi_{a}}{b} \Upsilon_{a}\right] \gamma_{1}=\left[\frac{\xi_{v}}{c}-i \frac{\xi_{a}}{b}\right] \gamma_{0}} & 4-43 b \\
{\left[\frac{\xi_{v}}{c} \Upsilon_{v}+\frac{\xi_{a}}{b} \Upsilon_{a}\right] \beta_{0}=\left[\frac{\xi_{v}}{c}+i \frac{\xi_{a}}{b}\right] \beta_{1}} & 4-43 c \\
{\left[\frac{\xi_{v}}{c} \Upsilon_{v}+\frac{\xi_{a}}{b} \Upsilon_{a}\right] \beta_{1}=\left[\frac{\xi_{v}}{c}-i \frac{\xi_{a}}{b}\right] \beta_{0}} & 4-43 d
\end{array}
$$

and

$$
\begin{array}{ll}
\gamma_{0}\left[\frac{\xi_{v}}{c} \Upsilon_{v}+\frac{\xi_{a}}{b} \Upsilon_{a}\right]=-\left[\frac{\xi_{v}}{c}+i \frac{\xi_{a}}{b}\right] \gamma_{1} & 4-44 a \\
\gamma_{1}\left[\frac{\xi_{v}}{c} \Upsilon_{v}+\frac{\xi_{a}}{b} \Upsilon_{a}\right]=-\left[\frac{\xi_{v}}{c}-i \frac{\xi_{a}}{b}\right] \gamma_{0} & 4-44 b \\
\beta_{0}\left[\frac{\xi_{v}}{c} \Upsilon_{v}+\frac{\xi_{a}}{b} \Upsilon_{a}\right]=-\left[\frac{\xi_{v}}{c}+i \frac{\xi_{a}}{b}\right] \beta_{1} & 4-44 c \\
\beta_{1}\left[\frac{\xi_{v}}{c} \Upsilon_{v}+\frac{\xi_{a}}{b} \Upsilon_{a}\right]=-\left[\frac{\xi_{v}}{c}-i \frac{\xi_{a}}{b}\right] \beta_{0} & 4-44 d
\end{array}
$$


The identities :

$$
\cosh (\|\xi\|)=\cosh ^{2}\left(\frac{1}{2}\|\xi\|\right)+\sinh ^{2}\left(\frac{1}{2}\|\xi\|\right) . \quad \sinh (\|\xi\|)=2 \sinh \left(\frac{1}{2}\|\xi\|\right) \cosh \left(\frac{1}{2}\|\xi\|\right) .
$$

must be used always in order to reproduce the $\cosh (\|\xi\|), \sinh (\| \xi||)$ from their halfvalues.

In order to determine the precise form of of the generators $\Upsilon_{v}, \Upsilon_{a}$ associated with velocity and acceleration-boosts we must recur to the physical interpretation of those transformations. The $U(1,1)$ subalgebra of the full $U(1,3)$ algebra has always a $U(1)$ phase piece. Thus we may focus on the $S U(1,1)$ subalgebra of $S U(1,3)$. Notice that $S U(1,1)$ is also a sub-algebra of the four-dim conformal algebra $S U(2,2)$ which is not the same as the $S U(1,3)$. However the $S U(1,1)$ shares similarities with the conformal algebra. In particular the latter four-dim conformal algebra has four conformal boosts transformations $K_{\mu}$, four translations generators $P_{\mu}$ as well as six Lorentz rotations and a dilation.

It has been known for some time that the the four-dim conformal algebra $S U(2,2)$ admits a Clifford-algebraic realization. In particular, $P_{\mu}$ and $K_{\mu}$ can be recast as $\gamma_{\mu}\left(1 \pm \gamma_{5}\right)$, the Lorentz generators as $\gamma_{\mu} \gamma_{\nu}$ and the dilations as $\gamma_{5}$ giving a total of 15 generators.

The velocity boosts in the $4 D$ Phase space must be a linear superposition of the following bi-vectors :

$$
\Upsilon_{v}=a_{1} \gamma_{0} \gamma_{1}+a_{2} \beta_{0} \beta_{1}+a_{3} \gamma_{0} \beta_{1}+a_{4} \gamma_{1} \beta_{0} \quad 4-46
$$

where the first two bivectors correspond to rotations in the $x_{0}-x_{1}$ and $p_{0}-p_{1}$ planes respectively and the last two terms are rotations mixing coordinates with momenta.

Inspired by the Clifford-realizations of the $P_{\mu}$ ( translation ) and $K_{\mu}$ (conformal boosts) generators we must have the following 8 bi-vectors in the definition of acceleration boosts in $4 D$ Phase-Space :

$$
\Upsilon_{a}=b_{ \pm}^{1} \gamma_{1}\left(1 \pm \gamma_{5}\right)+b_{ \pm}^{2} \beta_{1}\left(1 \pm \beta_{5}\right)+b_{ \pm}^{3} \beta_{1}\left(1 \pm \gamma_{5}\right)+b_{ \pm}^{4} \gamma_{1}\left(1 \pm \beta_{5}\right)
$$

The first four terms are linear combinations of the $P_{\mu}, K_{\mu}$ generators in the coordinates $x$-space and their dual generators $\tilde{P}_{\mu}, \tilde{K}_{\mu}$ in the momentum $p$-space. The last four terms represent the mixing of coordinates with momenta.

Despite having a total of $4+8=12$ coefficients in the definitions of $\Upsilon_{v}, \Upsilon_{a}$ we have a net number of 11 independent coefficient- ratios :

$$
a_{2} / a_{1}, \quad a_{3} / a_{1} . \quad a_{4} / a_{1} . \quad b_{+}^{1} / a_{1}, \quad b_{-}^{1} / b_{+}^{1}, \quad b_{ \pm}^{2} / b_{+}^{1}, \quad b_{ \pm}^{3} / b_{+}^{1}, \quad b_{ \pm}^{4} / b_{+}^{1} . \quad 4-48
$$

The ratio $b_{+}^{1} / a_{1}$ must be included since it represents the relative sign and magnitude among the $\Upsilon_{v}$ and $\Upsilon_{a}$ generators. One can scale off the coefficients $a_{1}, b_{1}$ out of the expressions and re-absorb them in the definitions of $\xi_{v} / c, \xi_{a} / b$ respectively, but there is a remaining relative \pm sign between the coefficients determining the $\Upsilon_{v}$ and $\Upsilon_{a}$ generators. Concluding, we have 11 independent ratios which match the number of $11=3+8$ 
condtions satisfied by the generators $\Upsilon_{v}, \Upsilon_{a}$ associated with velocity and accelerationboosts .

$$
\begin{aligned}
& \mathbf{Z}=x^{0} \gamma_{0}+p^{0} \beta_{0}+p^{1} \beta_{1}+x^{1} \gamma_{1} . \quad \mathbf{Z}^{\prime}=\mathbf{R} \mathbf{Z} \mathbf{R}^{-1} \Rightarrow \\
& <\mathbf{Z}^{\prime} \mathbf{Z}^{\prime}>_{\text {scalar }}=\left(x_{0}^{\prime}\right)^{2}+\left(p_{0}^{\prime}\right)^{2}-\left(x_{1}^{\prime}\right)^{2}-\left(p_{1}^{\prime}\right)^{2}=z_{0}^{\prime} \bar{z}_{0}^{\prime}-z_{1} \bar{z}_{1}^{\prime}=
\end{aligned}
$$

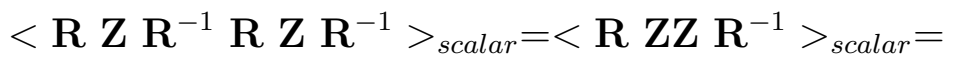

$$
\begin{aligned}
& <\mathbf{Z Z}>_{\text {scalar }}=\left(x_{0}\right)^{2}+\left(p_{0}\right)^{2}-\left(x_{1}\right)^{2}-\left(p_{1}\right)^{2}=z_{0} \bar{z}_{0}-z_{1} \bar{z}_{1}=\text { invariant. } \quad 4-49
\end{aligned}
$$

In the full fledged Clifford-Born-Phase-Spaces one requires taking the reversal operation $\tilde{\mathbf{Z}}$ by reversing the orderings of the $\gamma, \beta$ basis elements in the expansion of a polyvector in order to evaluate the inner product of two polyvectors : $\langle\tilde{\mathbf{Z}} \mathbf{Z}\rangle$. When $\mathbf{Z}$ is a vector there is no distinction between the reversal of $\mathbf{Z}$ and itself. Invariance holds provided $\mathbf{R}^{-1}=\tilde{\mathbf{R}}$ which restricts the form of the terms inside the exponential $\mathbf{R}=\exp \left[\theta^{M} E_{M}\right]$ when $E_{M}$ span the full graded structure of the Clifford algebra. For bivectors we have that the reversal of $\left(\gamma_{\mu} \gamma_{\nu}\right)$ is given by $\gamma_{\nu} \gamma_{\mu}=-\gamma_{\mu} \gamma_{\nu}$. Thus there is a sign reversal as expected in the terms inside the exponential in order to ensure us that $\tilde{\mathbf{R}}=\mathbf{R}^{-1}$.

Similar considerations follow for tri-vectors but not for 4 -vectors and 5-vectors basis elements. In the latter case the reversal operation does not change the sign. In order to remedy this situation one could construct a rotor of the form $\mathbf{R}=e^{i H}$ where $H$ is a Hermitian operator comprised of the $\theta^{M} E_{M}$ terms but now the $\theta^{M}$ are complex valued in general and the generators $E_{M}$ span the complexified Clifford algebra $C l(p, q) \oplus_{j} C l(p, q)$ - The inner products are defined now in terms of the Hermitian adjoint operation $\dagger$ $<\mathbf{Z}^{\dagger} \mathbf{Z}>$ where $\mathbf{Z}^{\dagger}=\tilde{\mathbf{Z}}^{*}$. In our case discussed above there is no problem with the reversal operation because the $\Upsilon_{v}, \Upsilon_{a}$ generators are defined in terms of bivectors which do change signs appropriately under the reversal operation and consequently $\tilde{\mathbf{R}}=\mathbf{R}^{-1}$.

To sum up, the velocity and acceleration/force boosts transformations acting on the $4 D$ Phase Space as :

$$
\begin{array}{ccc}
{\left[\cosh \left(\frac{1}{2}\|\xi\|\right)+\right.} & \left.\left(\frac{\xi_{v}}{c} \Upsilon_{v}+\frac{\xi_{a}}{b} \Upsilon_{a}\right) \frac{\sinh \left(\frac{1}{2}\|\xi\|\right)}{\|\xi\|}\right]\left[x^{0} \gamma_{0}+p^{0} \beta_{0}+x^{1} \gamma_{1}+p^{1} \beta_{1}\right] \times & \\
& {\left[\cosh \left(\frac{1}{2}\|\xi\|\right)-\left(\frac{\xi_{v}}{c} \Upsilon_{v}+\frac{\xi_{a}}{b} \Upsilon_{a}\right) \frac{\sinh \left(\frac{1}{2}\|\xi\|\right)}{\|\xi\|}\right] .} & 4-50
\end{array}
$$

Upon performing some straightforward algebra; introducing the $\mathcal{F}=b$ factors in the momenta terms ; recurring to the 11 conditions ( 3 orthonormality and 8 flip conditions which fix the 11 coefficient ratios ) and by defining $z_{0}=x_{0}+i p_{0} / b ; z_{1}=x_{1}+i p_{1} / b$, it leads to :

$$
\begin{array}{ll}
z_{1}^{\prime}=z_{1} \cosh (\|\xi\|)+z_{0}\left(\xi_{v} / c+i \xi_{a} / b\right) \frac{\sinh (\|\xi\|)}{\|\xi\|} & 4-50 b \\
z_{0}^{\prime}=z_{0} \cosh (\|\xi\|)+z_{1}\left(\xi_{v} / c-i \xi_{a} / b\right) \frac{\sinh (\|\xi\|)}{\|\xi\|} & 4-50 c
\end{array}
$$


and their complex conjugates, which are precisely the same transformations provided earlier for the $z_{0}, z_{1}, \bar{z}_{0}, \bar{z}_{1}$ variables. The laborious calculation is to solve for the values of the 11 coefficient ratios from the 11 conditions.

The ordinary $8 D$ Phase Space coordinates $x^{\mu}, p^{\mu}$ can be be written in terms of 4 complex coordinates $z_{0}, z_{1}, z_{2}, z_{3}$, by the standard procedure $z^{\mu}=x^{\mu}+i p^{\mu}$ and extended to Clifford-Phase-Space by :

$$
z^{\mu}=x^{\mu} \pm i p^{\mu}, \quad z^{\mu \nu}=x^{\mu \nu} \pm i p^{\mu \nu}, \quad z^{\mu \nu \rho}=x^{\mu \nu \rho} \pm i p^{\mu \nu \rho}, \ldots \ldots . \quad 4-50 d
$$

after absorbing the $L_{P}, R, \mathcal{F}$ factors in the definitions of the Clifford-Phase-Space coordinates. Notice, however, that the corresponding Clifford algebra associated with 4 complex dimensions ( 8 real dimensions of ordinary Phase space ) is the complexified Clifford algebra in four dimensions $C l_{C}(4)$ that can be realized in terms of the matrix algebra $\mathcal{M}(4, C)$ of $4 \times 4$ complex entries, but the latter matrix algebra $\mathcal{M}(4, C)$ with $2 \times 4 \times 4=32$ degrees of freedom is much smaller than the Quaternionc matrix algebra $\mathcal{M}(8, \mathbf{H})$ with $4 \times 8 \times 8=256$ degrees of freedom associated with the real $C l(2,6), C l(6,2)$ algebras. The latter Quaternionic matrix algebra is 8 times "bigger" than the former complex matrix algebra. There are two possibilities one can choose to study the symmetries of the Clifford-Phase-Space associated with an ordinary $8 D$ Phase space :

i. Work with the complex matrix algebra $\mathcal{M}(4, C)$ corresponding to the complexified Clifford $C l_{C}(4)$ algebra that is associated with 4 complex dimensions. Since complexified $4 D$ Minkowski spacetime is the arena for Twistors [74], this is a very appealing case to study.

ii. Work with the Quaternionic matrix $\mathcal{M}(8, \mathbf{H})$ algebra associated with the Real Clifford algebras $C l(2,6), C l(6,2)$. This case should be related to a Quaternionic and Octonionic ( if possible ) extensions of Twistor Theory .

To sum up, one can combine, simultaneously, the generalized velocity and acceleration/force boosts, and the additional symmetries acting in the full Cliffod-Phase-Space, by recurring to the matrix algebra $\mathcal{M}(8, \mathbf{H})$ realization of the $C l(2,6)$ algebra in order to implement the generalized poly-rotations of all the Clifford-valued holographic coordinates and momenta:

$$
x^{\mu, \nu}, \quad x^{\mu \nu \rho}, \ldots, \quad p^{\mu \nu}, \quad p^{\mu \nu \rho}, \ldots
$$

among each other. This geometrical picture is instrumental to be able to generalize these velocity and acceleration/force boosts transformations in Clifford-Phase-Spaces ( CPspaces ) that will reshuffle the holographic coordinates and holographic momenta among each other.

The full action of the Clifford group $C l(2,6)$ on the Clifford-Phase-Space variables is of the form :

$$
\mathbf{Z}^{\prime}=\mathbf{R} \mathbf{Z} \mathbf{R}^{-1}=e^{\theta^{A} E_{A}} Z^{M} E_{M} e^{-\theta^{A} E_{A}}
$$

$E_{A}$ runs over the full $C l(2,6)$ group :

$$
\gamma_{\mu_{1}} ; \gamma_{\mu_{1}} \wedge \gamma_{\mu_{2}} ; \ldots . . \quad \beta_{\nu_{1}} ; \beta_{\nu_{1}} \wedge \beta_{\nu_{2}} ; \ldots \quad \gamma_{\mu_{1}} \wedge \beta_{\nu_{1}}, \gamma_{\mu_{1}} \wedge \gamma_{\mu_{2}} \wedge \beta_{\nu_{1}}, \ldots . \quad 4-52
$$


The condition $<\tilde{\mathbf{Z}} \mathbf{Z}>=$ invariant is true if $\mathbf{R}^{-1}=\tilde{\mathbf{R}}$ which restricts the type of $E_{M}$ basis elements allowed in the definition of $\mathbf{R}$. A Unitary operator $U=e^{i H}$ for $H$ Hermitian requires the complexification of the $C l(2,6)$ algebra. In this case an invariant inner product $<\mathbf{Z}^{\dagger} \mathbf{Z}>$ can be constructed such $\mathbf{Z}^{\prime}=\mathbf{U} \mathbf{Z} \mathbf{U}^{\dagger}$.

\subsection{On the Noncommutative Yang's Spacetime Algebra, Holog- raphy and Area Quantization in Clifford Spaces}

We end this work with some relevants remarks about the impact of Yang's Noncommutative spacetime algebra on modified Newtonian dynamics. Such algebra involves two length scales, the minimal Planck scale $L_{P}=\lambda$ and an upper infrared cutoff scale $\mathcal{R}$.

Recently in [29] an isomorphism between Yang's Noncommutative space-time algebra (involving two length scales) [30] and the holographic area coordinates algebra of Cspaces (Clifford spaces) was constructed via an $A d S_{5}$ space-time (embedded in $6 D$ ) which is instrumental in explaining the origins of an extra (infrared) scale $\mathcal{R}$ in conjunction to the (ultraviolet) Planck scale $\lambda$ characteristic of C-spaces. Yang's Noncommutative spacetime algebra allowed Tanaka [31] to explain the origins behind the discrete nature of the spectrum for the spatial coordinates and spatial momenta which yields a minimum length-scale $\lambda$ (ultraviolet cutoff) and a minimum momentum $p=\hbar / \mathcal{R}$ ( maximal length $\mathcal{R}$, infrared cutoff ) .

Related to the issue of area-quantization, the norm-squared $\mathbf{A}^{2}$ of the holographic Area operator $X_{A B} X^{A B}$ in Clifford-spaces has a correspondence with the quadratic Casimir operator $\lambda^{4} \Sigma_{A B} \Sigma^{A B}$ of the conformal algebra $S O(4,2)(S O(5,1)$ in the Euclideanized $A d S_{5}$ case ). This holographic area-Casimir relationship does not differ much from the area-spin relation in Loop Quantum Gravity $\mathbf{A}^{2} \sim \lambda^{4} \sum j_{i}\left(j_{i}+1\right)$ in terms of the $S U(2)$ Casimir $J^{2}$ with eigenvalues $j(j+1)$, where the sum is taken over the spin network sites [28] and the minimal Planck scale emerges from a regularization procedure.

The Yang's algebra can be written in terms of the $6 D$ angular momentum operators and a $6 D$ pseudo-Euclidean metric $\eta^{M N}$ :

$$
\begin{aligned}
& \hat{M}^{\mu \nu}=\hbar \Sigma^{\mu \nu} . \quad \hat{M}^{56}=\hbar \Sigma^{56} . \\
& 4-53 \\
& \lambda \Sigma^{\mu 5}=\hat{x}^{\mu} . \quad \frac{\hbar}{\mathcal{R}} \Sigma^{\mu 6}=\hat{p}^{\mu} . \\
& 4-2 \\
& \mathcal{N}=\frac{\lambda}{\mathcal{R}} \Sigma^{56} . \\
& 4-54
\end{aligned}
$$

as follows :

$$
\begin{gathered}
{\left[\hat{p}^{\mu}, \mathcal{N}\right]=-i \eta^{66} \frac{\hbar}{R^{2}} \hat{x}^{\mu}} \\
{\left[\hat{x}^{\mu}, \mathcal{N}\right]=i \eta^{55} \frac{L_{P}^{2}}{\hbar} \hat{p}^{\mu}}
\end{gathered}
$$$$
4-56
$$ 


$$
\begin{array}{cc}
{\left[\hat{x}^{\mu}, \hat{x}^{\nu}\right]=-i \eta^{55} L_{P}^{2} \Sigma^{\mu \nu} .} & 4-57 \\
{\left[\hat{p}^{\mu}, \hat{p}^{\nu}\right]=-i \eta^{66} \frac{\hbar^{2}}{R^{2}} \Sigma^{\mu \nu} .} & 4-58 \\
{\left[\hat{x}^{\mu}, \hat{p}^{\mu}\right]=i \hbar \eta^{\mu \nu} \mathcal{N} .} & 4-59 \\
{\left[\hat{x}^{\mu}, \Sigma^{\nu \rho}\right]=\eta^{\mu \rho} x^{\nu}-\eta^{\mu \nu} x^{\rho} .} & 4-60 \\
{\left[\hat{p}^{\mu}, \Sigma^{\nu \rho}\right]=\eta^{\mu \rho} p^{\nu}-\eta^{\mu \nu} p^{\rho} .} & 4-61
\end{array}
$$

The dynamical consequences of the Yang's Noncommutative spacetime algebra can be derived from the quantum/classical correspondence :

$$
\frac{1}{i \hbar}[\hat{A}, \hat{B}] \leftrightarrow\{A, B\}_{P B} .62
$$

i.e. commutators correspond to Poisson brackets. More precisely, to Moyal brackets. in Phase Space. In the classical limit $\hbar \rightarrow 0$ Moyal brackets reduce to Poisson brackets. Since the coordinates and momenta are no longer commuting variables the classical Newtonian dynamics is going to be modified since the symplectic two-form $\omega^{\mu \nu}$ in Phase Space will have additional non-vanishing elements stemming from these non-commuting coordinates and momenta.

In particular, the modified brackets read now :

$$
\begin{gathered}
\{\{A(x, p), B(x, p)\}\}=\partial_{\mu} A \omega^{\mu \nu} \partial_{\nu} B=\{A(x, p), B(x, p)\}_{P B}\left\{x^{\mu}, p^{\nu}\right\}+ \\
\frac{\partial A}{\partial x^{\mu}} \frac{\partial B}{\partial x^{\nu}}\left\{x^{\mu}, x^{\nu}\right\}+\frac{\partial A}{\partial p^{\mu}} \frac{\partial B}{\partial p^{\nu}}\left\{p^{\mu}, p^{\nu}\right\} .
\end{gathered}
$$

If the coordinates and momenta were commuting variables the modified bracket will reduce to the first term only :

$$
\{\{A(x, p), B(x, p)\}\}=\{A(x, p), B(x, p)\}_{P B}\left\{x^{\mu}, p^{\nu}\right\}=\left[\frac{\partial A}{\partial x^{\mu}} \frac{\partial B}{\partial p^{\nu}}-\frac{\partial A}{\partial p^{\mu}} \frac{\partial B}{\partial x^{\nu}}\right] \eta^{\mu \nu} \mathcal{N}
$$

The ordinary Heisenberg ( canonical ) algebra is recovered when $\mathcal{N} \rightarrow 1$ in eq-(4-13).

In the nonrelativistic limit, the modfied dynamical equations are :

$$
\begin{gathered}
\frac{d x^{i}}{d t}=\left\{\left\{x^{i}, H\right\}\right\}=\frac{\partial H}{\partial p^{j}}\left\{x^{i}, p^{j}\right\}+\frac{\partial H}{\partial x^{j}}\left\{x^{i}, x^{j}\right\} . \\
\frac{d p^{i}}{d t}=\left\{\left\{p^{i}, H\right\}\right\}=-\frac{\partial H}{\partial x^{j}}\left\{x^{i}, p^{j}\right\}+\frac{\partial H}{\partial p^{j}}\left\{p^{i}, p^{j}\right\} .
\end{gathered}
$$

The non-relativistic Hamiltonian for a central potential $V(r)$ is : 


$$
H=\frac{p_{i} p^{i}}{2 m}+V(r) . \quad r=\left[\sum_{i} x_{i} x^{i}\right]^{1 / 2}
$$

Defining the magnitude of the central force by $F=-\frac{\partial V}{\partial r}$ and using $\frac{\partial r}{\partial x^{i}}=\frac{x_{i}}{r}$ one has the modified dynamical equations of motion :

$$
\begin{array}{cc}
\frac{d x^{i}}{d t}=\left\{\left\{x^{i}, H\right\}\right\}=\frac{p_{j}}{m} \delta^{i j}-F \frac{x_{j}}{r} L_{P}^{2} \Sigma^{i j} . & 4-68 \\
\frac{d p^{i}}{d t}=\left\{\left\{p^{i}, H\right\}\right\}=F \frac{x_{j}}{r} \delta^{i j}+\frac{p_{j}}{m} \frac{\Sigma^{i j}}{R^{2}} . & 4-69
\end{array}
$$

The angular momentum two-vector $\Sigma^{i j}$ can be written as the dual of a vector $\vec{J}$ as follows $\Sigma^{i j}=\epsilon^{i j k} J_{k}$ so that :

$$
\begin{array}{cc}
\frac{d x^{i}}{d t}=\left\{\left\{x^{i}, H\right\}\right\}=\frac{p^{i}}{m}-L_{P}^{2} F \frac{x_{j}}{r} \epsilon^{i j k} J_{k} . & 4-70 a \\
\frac{d p^{i}}{d t}=\left\{\left\{p^{i}, H\right\}\right\}=F \frac{x^{i}}{r}+\frac{p_{j}}{m} \frac{\epsilon^{i j k} J_{k}}{R^{2}} & 4-70 b
\end{array}
$$

For planar motion ( central forces ) the cross-product of $\vec{J}$ with $\vec{p}$ and $\vec{x}$ is not zero since $\vec{J}$ points in the perpendicular direction to the plane. Thus, one will have nontrivial corrections to the ordinary Newtonian equations of motion induced from Yang's Noncommutative spacetime algebra in the non-relativistic limit. When $\vec{J}=0$, pure radial motion, there are no corrections. This is not the case when we studied the modified Newtonian dynamics in the previous section of the modified Schwarzschild field due to the maximal-acceleration relativistic effects. Therefore, the two routes to obtain modifications of Newtonian dynamics are very different.

Concluding, eqs-(4-68, 4-69, 4-70a , 4-70b ) determine the modified Newtonian dynamics of a test particle under the influence of a central potential explicitly in terms of the two $L_{P}, R$ minimal/maximal scales. When $L_{P} \rightarrow 0$ and $R \rightarrow \infty$ one recovers the ordinary Newtonian dynamics $v^{i}=\left(p^{i} / m\right)$ and $F\left(x^{i} / r\right)=m\left(d v^{i} / d t\right)$. The unit vector in the radial direction has for components $\hat{r}=(\vec{r} / r)=\left(x^{1} / r, x^{2} / r, x^{3} / r\right)$.

It is warranted to study the full relativistic dynamics as well, in particular the modified relativistic dynamics of the de-Sitter rigid top [41] due to the effects of Yang's Noncommutative spacetime algebra . The de Sitter rigid Top can be generalized further to Clifford spaces since a Clifford-polyparticle has more degrees of freedom than a relativistic top in ordinary spacetimes [40] . And, naturally, to study the modified Nambu-Poisson dynamics of p-branes [43] as well. 


\section{Gravity and the Standard Model from a $\mathrm{Cl}(1,7)$ Group Graded Structure}

We will follow very closely the main results of Smith [32] to get a representation of all the known particles and fields in Physics based on the real Clifford group $C l(1,7)$ ( one timelike and 7 spacelike directions ). The $C l(1,7)$ is $2^{8}=256=16 \times 16$ dimensional and has a graded structure :

$$
\begin{array}{llllllllll}
1 & 8 & 28 & 56 & 35+35 & 56 & 28 & 8 & 1 & 5-1
\end{array}
$$

into a scalar, vector, bivector, 3 -vector, ...... pseudoscalar. The middle 70 is written as $35+35$ because it is self-dual under Hodge duality. By Hodge duality, the 182856 35 is dual to the 35562881 . Marks ${ }^{1}$ has shown that the 18285635 correspond to physical fields in the coordinate representation while the 35562881 correspond to physical fields in the momentum representation and complementarity between space-time and momentum-energy is achieved by bit inversion, which interconverts between position representation and momentum representation. The model [32] does use all the graded parts of $\mathrm{Cl}(8)$, and also the spinor structure of $\mathrm{Cl}(8)$, but the 56 and 35 parts are not physically effective at low energies after dimensional reduction, and consequently they are not written down explicitly in the $8 D$ Lagrangian below which is used to calculate force strengths, particle masses, etc in the low energy region where we do experiments today.

\subsection{Spinors as left/right ideal elements of Clifford Algebras}

Spinors are related to the graded structure of 256-dim $\mathrm{Cl}(8),{ }^{2}$ Lounesto discusses [4] that in order to fix a minimal left ideal $\mathrm{V}$ of $\mathrm{Cl}(\mathrm{p}, \mathrm{q})$ we can choose a primitive idempotent $f$ of $\mathrm{Cl}(\mathrm{p}, \mathrm{q})$ so that $V=C l(p, q) f$. By means of an orthonormal basis $\left\{e_{1}, e_{2}, \ldots, e_{n}\right\}$ for the grade-1 vector part of $C l(p, q)$ represented as $C l^{1}(p, q)$ we can construct a primitive idempotent $f$ as follows: Recall that the $2^{n}$ elements

$$
e_{A}=e_{a_{1}} e_{a_{2}} \ldots e_{a_{k}}, \quad 1<a_{1}<a_{2}<\ldots<s_{k}<n . \quad 5-2
$$

constitute a basis for $C l(p, q)$ with $\operatorname{dim}_{R} V=2^{X}$, where $X=h$ or $X=h+1$ according as $p-q=0,1,2 \bmod 8$ or $p-q=3,4,5,6,7 \bmod 8$ and $h=[n / 2]$. Select $n-X$ elements $e_{A}, e_{A}^{2}=1$, so they are pairwise commuting and generate a group of order $2^{n-X}$. then the idempotent

$$
f=(1 / 2)\left(1+e_{A_{1}}\right)(1 / 2)\left(1+e_{A_{2}}\right) \ldots(1 / 2)\left(1+e_{A_{(n-X)}}\right) .
$$

is primitive . In particular, $C l(1,7)$ has a primitive idempotent comprised of $1+7+7+1=$ 16 elements :

$$
f=(1 / 2)\left(1+e_{1248}\right)(1 / 2)\left(1+e_{2358}\right)(1 / 2)\left(1+e_{3468}\right)(1 / 2)\left(1+e_{4578}\right)=
$$

\footnotetext{
${ }^{1}$ http://www.innerx.net/personal/tsmith/clfpq2.html clifstructure

${ }^{2}$ http://www.innerx.net/personal/tsmith/8idempotents.html
} 


$$
\begin{gathered}
(1 / 16)\left(1+e_{1248}+e_{2358}+e_{3468}+e_{4578}+e_{5618}+e_{6728}+e_{7138}-\right. \\
\left.e_{3567}-e_{4671}-e_{5712}-e_{6123}-e_{7234}-e_{1345}-e_{2456}+e_{J}\right) .
\end{gathered}
$$

with four factors, where $J=12345678$ so $e_{J}^{2}=-1$.

Hence, the spinor has a mixed graded structure comprised of a scalar, a pseudoscalar, 74 -vectors and their 7 dual 4 -vectors. These 16 elements (terms) necessary to extract (to read-off) a spinor from the full Clifford multi-graded structure match precisely the number of components of a spinor in $D=8$ given by $2^{8 / 2}=16$. Since primitive idempotents produces spinors, the Higgs and spinors are connected, which gives a $\mathrm{Cl}(8)$ structure interpretation for the Yukawa couplings, something that is not clearly motivated in the usual Standard Model. In short, the spinors in the model [32] stem from a primitive idempotent living in the scalar, the $35+354$-vector and pseudoscalar parts of the $\mathrm{Cl}(8)$ graded structure.

A way to look at superspace in terms of Clifford algebras is described by Doran, Lasenby and Gull [79] where given a set of $n$ Grassmann generators one can map these into a Clifford geometric algebra by introducing a set of $n$ independent vectors and replacing the product of Grassmann variables by the exterior product. In this way any combination of Grassmann variables can be replaced by a multivector ( polyvector ) and the Berezin calculus can be handled entirely within the algebra by introducing the reciprocal frame. Integration is defined to be equivalent to right differentiation. Thus we see that Grassmann calculus amounts to no more than Clifford contraction and the results of "Grassmann analysis" can all be expressed as simple algebraic identities for multivectors (polyvectors) . Furthermore these results are now given a firm geometric significance through the identification of Clifford elements with directed line, plane segments etc.

A subtle "supersymmetry" exists in the 8-dim structure of Smith's model [32] , whereby the 28 gauge bosons are the $8 \wedge 8=28$ bivectors and there are $8_{+}$spinor fermion particles and $8_{-}$spinor fermion antiparticles. Due to triality, the vector $8_{v} \sim 8_{+} \sim 8_{-}$ so that one can relate the 28 bosons with the $\wedge$ product of the 8 fermions with the 8 antifermions. In other words, the subtle "supersymmetry" of the model [32] displays the nice features of 11 supergravity but does not have its unrealistic particle content, at low energies, of 128 bosonic degrees of freedom $\left(g_{\mu \nu}, A_{\mu \nu \rho}\right)$ and 128 fermionic degrees of freedom associated with the spin $3 / 2$ gravitino $\Psi_{\mu}$.

\subsection{The Standard Model and Gravity from 8D Clifford Struc- tures}

We will review in detail the derivation of all the Standard model parameters ( including neutrino masses ) from first principles by Smith [32]. Beck [33] has a derivation of all the Standard Model parameters including neutrino masses based on the Chaotic Quantization (Parisi-Wu) method associated with a Kaneko coupled-map two-dim lattice and iterated Ulam-Tschebysheff maps (symbolic dynamics). The Standard Model parameters appear as numerical minima ( and zeros ) of certain potentials. In Smith's model [32] they 
appear in terms of closed analytical geometrical expressions given by ratios of volumes in homogenous complex domains with Shilov boundaries, for example.

The features of Smith's model [32] are :

- The 1-dim scalar representation is associated with the Higgs scalar.

- The emergence of right/left handed chirality spinors and $\mathbf{3}$ generations.

As we have discussed, spinors correspond to left and right ideal elements of a Clifford algebra and can be visualized as columns or rows of a $16 \times 16$ matrix. In the model [32] there are two mirror-image spinors of opposite chirality, each of the form of a real $(1,7)$ column vector, that correspond to the first generation of fermion particles plus their anti-particles, with octonionic structure. The 8 fermions correspond to a neutrino, an electron, a red, blue, green up quark, and a red, blue, green down quark. The 8 mirror image fermions correspond to an anti-neutrino, a positron, a red, blue, green up anti-quark, and a red, blue, green down anti-quark.

Second and third generation of spinors emerge from the dimensional reduction of the $8 D$ spacetime so that the first generation is associated with octonions; the second generation with pairs of octonions and the third generations with triples of octonions which explains the different values of the constituent quark masses.

$\mathrm{Cl}(8)$ has exceptional, unusual symmetries ( the bi-vector Spin ( 8 ) exhibits a triality automorphism between spacetime vectors and spinors ) and structure, and is also a fundamental building block of all real Clifford algebras, due to real 8-periodicity. For instance, if one were to look at $\mathrm{Cl}(16)=\mathrm{Cl}(2 \mathrm{x} 8)=\mathrm{Cl}(8)$ (x) $\mathrm{Cl}(8)$ one would see a 2-level tensornesting of the $16 \times 16$ structure, and the $\mathrm{Cl}(16)$ diagonal would be made up of 16 terms, each of which looked like an entire $\mathrm{Cl}(8) 16 \times 16$, with its 16-element diagonal, so that $\mathrm{Cl}(16)$ spinors would look like $16 \times 16=256$ elements, with 128 chiral spinors and 128 spinors of opposite chirality, which is indeed the case.

- The correct $4 D$ spacetime signature $(1,3)$.

The model [32] is also consistent with the quaternionic structure of conformal $\mathrm{Cl}(2,4)$ $=4 \times 4$ quaternionic matrices and with the quaternionic structure of $\mathrm{Cl}(1,3)=2 \times 2$ quaternionic matrices, so the 4-dim physical spacetime has the correct signature $(1,3)$ and not the signature $(3,1)$ of $\mathrm{Cl}(3,1)=4 \mathrm{x} 4$ real matrices. Hence, the $(1,7)$-dimensional vector representation corresponds to an 8-dim high-energy spacetime with octonionic structure that reduces at lower energies to quaternionc structures that correspond to the $(1,3)$-dim physical spacetime and a $(0,4)$-internal symmetry space.

- Emergence of Gravity and $S U(3) \otimes S U(2) \otimes U(1)$.

There is a 28 -dim bivector representation $(28=16+12)$ that corresponds to the gauge symmetry Lie algebra of $\operatorname{Spin}(1,7)$ that reduces at lower energies to ( $\mathbf{i}$ ) a 16-dim $U(2,2)=U(1) \otimes S U(2,2)=U(1) \otimes \operatorname{Spin}(2,4)$ whose conformal Lie algebra structure leads to gravity (with a cosmological constant ) via the MacDowell-MansouriChamseddine-West mechanism, and ( ii ) a 12-dim $S U(3) \otimes S U(2) \otimes U(1)$ Standard Model symmetry group involving 12 Gauge Bosons ( 8 gluons, 3 weak bosons $W_{ \pm}, Z_{0}$, and the photon) that can be represented on an internal 4-dim symmetry space by the coset structure $S U(3) / U(2)=S U(3) / S U(2) \otimes U(1)$ associated with a $C P^{2}$ projective space. 
- Cosmological implications.

The model also gives two significant sets of calculations which are : (i) - WMAP ratio calculation ${ }^{3}$ and (ii) - neutrino mass and mixing angle calculations ${ }^{4}$

- Unified Lagrangian in $8 D$.

The above structures fit together into an $8 D$ ( $4 D$ spacetime with a $4 D$ internal symmetry space ) Lagrangian :

$$
\int_{V_{8}} F \wedge{ }^{*} F++\bar{\Psi} \mathbf{d} \Psi+(d \Phi+[A, \Phi]) \wedge{ }^{*}(d \Phi+[A, \Phi]) . \quad 5-5
$$

$\bar{\Psi}=\Psi^{\dagger} \Gamma_{0}$ and $\mathbf{d}=\Gamma^{M} \partial_{M}$. that reduces to the Lagrangian of Gravity plus the Standard Model upon dimensional reduction as shown in [32]

- Hermitian Symmetric Spaces.

The geometry of these representation spaces is associated with complex homogeneous domains with Shilov boundaries. In conjunction with the combinatorial structure of the second and third generation fermions (based on paths along the internal dimensions) allows the explicit calculation and derivation of the relative force strength of all coupling constants and particle masses, The coupling constants are defined as the probability of a "charged" particle to emit a gauge boson. The mass $m$ is defined as the probablity for a particle to change direction. Based on these physical definitions Smith [32] was able to derive the following :

- The EM fine structure constant 1/137.03608 at the characteristic energy of the Bohr radius scale.

- A Fermi coupling of 0,235 at a characteristic energy of $100 \mathrm{Gev} ; g_{\text {weak }} m_{\text {proton }}^{2}$ is about $1.02 \times 10^{-5}$.

A scalar Higgs particle of mass $m_{H}=146 \mathrm{Gev}$ and a vev of $252.5 \mathrm{Gev}$.

$W_{ \pm}$masses $m_{W}=80.326 \mathrm{Gev}$ and the $Z_{0}$ mass $m_{Z}=91.862 \mathrm{Gev}$. The Weinberg angle $\sin ^{2} \theta_{W}=1-\left(m_{W} / m_{Z}\right)=0.235$.

- The running color force strength couplings : 0.6286, 0.167, 0.121, 0.106 at 0.245 Gev, 5.3 Gev, 34 Gev, 91 Gev respectively.

- the gravitational coupling $G_{N}$ such $G_{N} m_{\text {proton }}^{2}=5 \times 10^{-39}$.

- electron mass $m_{e}=0.511 \mathrm{Mev}$. Up and Down constituent quark mass $m_{u}=m_{d}=$ $312.8 \mathrm{Mev}$.

- Muon mas $m_{\mu}=104.8 \mathrm{Mev}$. Strange and Charm constituent quarks of mass $m_{s}=625 \mathrm{Mev}$ and $m_{c}=2.09 \mathrm{Gev}$.

- Tau mass $m_{\tau}=1.88 \mathrm{Gev}$. Bottom quark constituent mass $m_{b}=5.63 \mathrm{Gev}$ and a top (truth) quark ) of mass 130 Gev consistent with the analysis of the Fermilab data by Dalitz and Goldstein [58]. The 170 Gev consensus value for the top ( truth ) quark is associated with a 3 -jet interpretation of the 170 Gev peaks, whereas the 2-jet interpretation supports a $130-140$ Gev mass analysis for the top ( truth ) quark. Dileptonic events, being more directly measured with fewer jets, give a relatively accurate top ( truth ) quark mass.

\footnotetext{
${ }^{3}$ http://www.innerx.net/personal/tsmith/coscongraviton.html

${ }^{4}$ http://www.innerx.net/personal/tsmith/snucalc.html asno
} 
Although there are more semileptonic events, they involve background models that use complicated calculations of electroweak and QCD processes that may not yet be fully understood. $6 \pi^{5}$.

- Proton mass $m_{p}=938.25 \mathrm{Mev}$ and proton to electron mass ratio : $m_{p} / m_{e}=1836 \sim$

- Neutrino masses, mixing angle calculations, Yukawa couplings and CabibboKobayashi-Maskawa quark mixing parameters ${ }^{5}$

\subsection{On the Interplay among Clifford, Division, Jordan and Ex- ceptional Lie algebras with $\mathrm{D}=26,27,28$ dimensions}

Roughly speaking, we can interpret $D=26,27,28$ dimensions as the 3 -foldings of $8 D$ with strings, membranes and three- branes living transver sely to these 3 -folds $24=3 \times 8$ ; i.e. the number of transverse degrees for the strings, membranes and three -branes are 24 in all of these cases. The world-manifolds of a string, brane and three-brane are two, three and four-dimensional respectively. Hence, a bosonic and/or supersymmetric String, $\mathrm{M}, \mathrm{F}$ theory correspond to the following dimensions :

$8 k+2, D=10$ superstring for $k=1$ and $D=26$ Bosonic string for $k=3$. $8 k+3, D=$ $11 \mathrm{M}$ theory for $k=1$ and $D=27$ Bosonic membrane for $k=3.8 k+4, D=12 \mathrm{~F}$ theory for $k=1$ and $D=28$ Bosonic 3-brane for $k=3$. The interplay among $D=26,27,28$ dimensions as explained by Smith goes as follows.

The 28-real-dimensional degree-4 quaternionic Jordan algebra J4(Q) of 4x4 Hermitian matrices over the Quaternions ${ }^{6}$

$p D B A$

$D^{*} q E C$

$B^{*} E^{*} r F$

$A^{*} C^{*} F^{*} t$

where ${ }^{*}$ denotes conjugate and p,q,r,t are in the reals $\mathrm{R}$ and $\mathrm{A}, \mathrm{B}, \mathrm{C}, \mathrm{D}, \mathrm{E}, \mathrm{F}$ are in the quaternions $Q=\mathbf{H}$. The $4 \times 28=112$-real dimensional Quaternification of J4(Q) can be represented as the Symmetric Space $E_{8} / E_{7} \times S U(2)$. J4(Q) contains the traceless 28-1 $=27$-dimensional subalgebra J4(Q)o that has the unique structure of the 27-dimensional exceptional Jordan algebra $J 3(O)$ of $3 \times 3$ Hermitian matrices over the Octonions

$p B A$

$B^{*} q C$

$A^{*} C * r$

where ${ }^{*}$ denotes conjugate and p,q,r are in the reals $\mathrm{R}$ and $\mathrm{A}, \mathrm{B}, \mathrm{C}$ are in the Octonions O. The $2 \times 27=54$-real dimensional Complexification of $J 3(O)=J 4(Q) o$ can be represented as the Symmetric Space $E_{7} / E_{6} \times U(1)$. J3(O) contains a traceless 27-1 $=26$-dimensional subalgebra $J 3(O) o$ that can be represented as the Symmetric Space $E_{6} / F_{4}$.

\footnotetext{
${ }^{5}$ http://www.innerx.net/personal/tsmith/snucalc.html asno

${ }^{6}$ http://www.innerx.net/personal/tsmith/Jordan.html
} 
In other words, the chain of dimensions $D=26,27,28$ yields Jordan algebra structures $J 3(O) o, J 3(O)=J 4(Q) o, J 4(Q)$ which in turn furnish the Lie algebra structures $E_{6}, E_{7}, E_{8}$.

To connect with (real) Clifford algebras, i.e. how to fit $E_{8}$ into a Clifford structure , start with the 248-dim $E_{8}=120$-dim bivector adjoint of D8 + 128-dim D8 half-spinor and so embed $E_{8}$ in the Clifford algebra $\mathrm{Cl}(16)$, with graded structure

\section{0}

$$
11440800843681820560120161
$$

and total dimension $2^{16}=65,536=(128+128)(128+128)$

Since $\mathrm{Cl}(16)=\mathrm{Cl}(2 \times 8)=\mathrm{Cl}(8) \mathrm{xCl}(8)$, in particular, $120=1 \times 28+8 \times 8+28 \times 1$ and $128=8 \times 8+8 \times 8$, hence $248=120+128$ and $E_{8}$ can be represented in a tensor product of $\mathrm{Cl}(8)$ algebras.

Other important topics discussed in Smith's website are :

- Freudenthal-Tits Magic Square

- Algebraic Geometry....Compactifications

- The Monster Group

- Algebraic Geometry

- Projective Geometry and Clifford Geometric Calculus

- Coxeter $A_{k}, D_{k}, E_{6}, E_{7}, E_{8}$ Singularities as Branchings among the Worlds of the Many-Worlds

- Infinite Dimensional Clifford Algebras, $E_{8}$ lattices and von Neumann Hyperfinite II1 Factors.

The global structure of physics could be described by many copies of $E_{8}$, or equivalently by a very large Clifford algebra so that the $C l(8)$ building blocks are consistently connected with each other so that their 8-dim vector spaces fit together to form a large $E_{8}$ lattice. We may recall that the conventional von Neuman Hyperfinite II1 factor is roughly an infinitedimensional version of the spinor representation of Complex Clifford algebras, which have periodicity 2 and so are like an infinite limit of what Baez calls "... the fermionic Fock space over $C^{(2 n)} \ldots$. " and then generalize it to the case of Real Clifford Algebras with periodicity 8 so that one gets is an infinite limit of a tensor product of a lot of copies of 256-dim $\mathrm{Cl}(8),{ }^{7}$

Each $\mathrm{Cl}(8)$ would describe physics locally in the neighborhood of a given spacetime point, as described in the physics model ${ }^{8} \mathrm{All}$ the $\mathrm{Cl}(8)$ factors in the generalized Hyperfinite II1 factor (roughly an infinite tensor product) would be linked together to form (at the next higher energy level above our quaternionic 4-dim physical spacetime plus 4-dim $C P^{2}$ internal symmetry space) a higher-energy real/octonionic 8-dim spacetime as described in ${ }^{9}$

\footnotetext{
${ }^{7}$ http://www.innerx.net/personal/tsmith/II1vNfactor.html

${ }^{8}$ http://www.innerx.net/personal/tsmith/2002SESAPS.html

${ }^{9}$ http://www.innerx.net/personal/tsmith/ClifTensorGeom.html
} 
When one takes quantum superpositions in the many-worlds quantum theory, quantum loops/graphs of higher and higher order appear, whose description involves the prime numbers ${ }^{10}$ and which may be closely related to the p-adic geometry.

For additional references pertaining the topics discussed in this section see [49] , [50] , [51] , [52], [53], [54], [55], [56], [57], [?].

\section{$6 \quad$ A Clifford Geometric Unified Action of all Forces}

Having outlined in detail Smith's model [32] and the important applications of Clifford algebras in the last section that yields numerical results which agree with experimental observations at low energies with high precision, the goal now is to embed Smith's model into the full-fledged geometrical structure consistent with the Extended Relativity Theory in Clifford-Phase spaces described in section 4 . At smaller scales of the order of the Planck length (at very high energies) the whole picture changes dramatically because the holographic coordinates and holographic momenta degrees of freedom in Clifford-Phase spaces are no longer suppressed and, instead, the full Clifford-Phase-Space Extended Relativistic dynamics sets in with a minimal Planck $L_{P}$ and upper length scales $R$ (minimum momentum). An ultraviolet and infrared cutoffs are built into the theory. Namely, we will no longer be dealing with point particles and fields, but with extended-objects ( $\mathrm{p}$ loops, closed p-branes) and their corresponding p-loop ( p-branes ) fields with much larger symmetries and degrees of freedom.

\subsection{Maxwell-Yang-Mills actions in Clifford Spaces}

In this sub-section we will review briefly the extension of Maxwell's theory of Electrodynamics associated with ordinary point-charges to a Generalized Maxwell theory in Clifford spaces involving extended charges and $p$-forms of arbitrary rank [70]

The purpose of studying Clifford-space extensions of Maxwell-Yang-Mills types of actions

$$
S=\int F_{\mu \nu} F^{\mu \nu} . \quad F_{\mu \nu}=\partial_{\mu} A_{\nu}-\partial_{\nu} A_{\mu}+\left[A_{\mu}, A_{\nu}\right]
$$

is because Smith's $8 D$ action based on all the multi-graded field components associated with the $C l(1,7)$ algebra : scalars, vectors, bivectors, trivectors, 4 -vectors, ..... discussed in detail in the previous section, could be embedded into more general actions of the type FF in Clifford-spaces.

The Clifford-valued gauge field $A(X)=A^{M}(X) E_{M}$ is now a function of the Cliffordpolyvector-valued coordinates $X=X^{M} E_{M}$. For example in $8 D$ one has $2^{8}=256$ degrees of freedom belonging to the polyvector coordinates :

$$
X=\Omega \mathbf{1}+x^{\mu} \Gamma_{\mu}+x^{\mu \nu} \Gamma_{\mu} \wedge \Gamma_{\nu}+x^{\mu \nu \rho} \Gamma_{\mu} \wedge \Gamma_{\nu} \wedge \Gamma_{\rho}+\ldots \ldots
$$

where we omitted suitable powers of the Planck scale $L_{P}$ in order to match dimensions.

\footnotetext{
${ }^{10}$ http://www.innerx.net/personal/tsmith/Rzetazeta.html qsohprime
} 
Secondly, the differential operator in C-spaces is now a generalized Dirac operator:

$$
d=E^{M} \partial_{M}=1 \partial_{\Omega}+\Gamma^{\mu} \partial_{x_{\mu}}+\Gamma^{\mu} \wedge \Gamma^{\nu} \partial_{x_{\mu \nu}}+\ldots
$$

the indices $M, N \ldots$ run over all the polyvector basis elements and range from $1,2 \ldots .2^{D}$ since a Clifford algebra in $D$-dim has $2^{D}$ basis elements. It is convenient to order the polyvector collective indices indices $M, N \ldots$ as :

$$
\mu_{1}<\mu_{2}<\mu_{3}<\ldots \ldots<\mu_{M} . \quad \nu_{1}<\nu_{2}<\nu_{3}<\ldots \ldots<\nu_{M} .
$$

Thirdly, the generalized Maxwell field strength in C-space is :

$$
\begin{aligned}
F= & d A=E^{M} \partial_{M}\left(E^{N} A_{N}\right)=E^{M} E^{N} \partial_{M} A_{N}=\frac{1}{2}\left\{E^{M}, E^{N}\right\} \partial_{M} A_{N}+ \\
& \frac{1}{2}\left[E^{M}, E^{N}\right] \partial_{M} A_{N}=\frac{1}{4} F_{(M N)}\left\{E^{M}, E^{N}\right\}+\frac{1}{4} F_{[M N]}\left[E^{M}, E^{N}\right] .
\end{aligned}
$$

where one has decomposed the Field strength components into a symmetric plus antisymmetric piece by simply writing the Clifford geometric product of two polyvectors $E^{M} E^{N}$ as the sum of an anticommutator plus a commutator piece respectively .

$$
\begin{gathered}
F_{(M N)}=\frac{1}{2}\left(\partial_{M} A_{N}+\partial_{N} A_{M}\right) . \\
F_{[M N]}=\frac{1}{2}\left(\partial_{M} A_{N}-\partial_{N} A_{M}\right) .
\end{gathered}
$$

A measure of integration in C-space can be written as:

$$
[\mathcal{D} X]=(d \Omega)\left(d x^{0} d x^{1} \ldots d x^{D}\right)\left(d x^{01} d x^{02} \ldots\right)\left(d x^{012} \ldots\right) \ldots \ldots\left(d x^{0123 \ldots D}\right) .
$$

The standard C-space Maxwell action is up to a numerical factor given by :

$$
S(A)=\int[\mathcal{D} X] F_{[M N]} F^{[M N]} .
$$

and is automatically gauge invariant under the transformations $\delta A_{M}=\partial_{M} \Lambda$ since $\delta F_{[M N]}=0$ due to the antisymmetry condition $\left[\partial_{M}, \partial_{N}\right] \Lambda=0$.

The action $[70],[1]$

$$
\int[\mathcal{D} X]<F^{\dagger} F>_{\text {scalar }}=\int[\mathcal{D} X]\left(a F_{(M N)} F^{(M N)}+b F_{[M N]} F^{[M N]}\right) .
$$

where $a, b$ are dimension-dependent coefficients, is invariant ( up to total derivatives) under infinitesimal gauge transformations provided the symmetric part of $F$ is divergence-free $\partial_{M} F^{(M N)}=0$

It was later noticed in [1] that this divergence-free condition has the same effects as if one were fixing a gauge leaving a residual symmetry of restricted gauge transformations such that the gauge symmetry parameter obeys the Laplace-like equation $\partial_{M} \partial^{M} \Lambda=$ 0 . Such residual ( restricted) symmetries are precisely those that leave invariant the divergence-free condition on the symmetric part of $F$. Residual, restricted symmetries 
occur, for example, in the light-cone gauge of p-brane actions leaving a residual symmetry of volume-preserving diffs and in string theory, in the conformal gauge, leaving a residual symmetry under conformal reparametrizations; i.e. the Virasoro algebras whose symmetry transformations are given by holomorphic and anti-holomorphic reparametrizations of the string world-sheet.

This Laplace-like condition on the gauge parameter is also the one required such that the action (6-8) is invariant under finite (restricted) gauge transformations since under such (restricted) finite transformations the Lagrangian changes by second-order terms of the form $\left(\partial_{M} \partial_{N} \Lambda\right)^{2}$, which are total derivatives if, and only if, the gauge parameter is restricted to obey the analog of Laplace equation $\partial_{M} \partial^{M} \Lambda=0$ Concluding, the effects of adding the symmetric pieces of $F$ to the action leads to a restricted gauge symmetry which has the same effects as if one were fixing a gauge $\partial_{M} F^{(M N)}=0$

The matter-field minimal coupling is :

$$
\int A_{M} d X^{M}=\int[\mathcal{D} X] A_{M} J^{M}
$$

where one has re-absorbed the coupling constant, the C-space analog of the electric charge, within the expression for the $A$ field itself and the current $J$ polyvector is proportional to the polymomentum, like in ordinary EM the current four-vector $J^{\mu}$ is proportional to the four-velocity with a delta function proportionality factor:

$$
\delta\left(x^{0}-x^{0}(\tau)\right) \delta\left(x^{1}-x^{1}(\tau)\right) \delta\left(x^{2}-x^{2}(\tau)\right) \delta\left(x^{3}-x^{3}(\tau)\right) .
$$

which implies that the current has support along the worldline of the particle $x^{\mu}=x^{\mu}(\tau)$.

This matter-field coupling term (6-9) has the same form as the coupling of p-branes (whose world volume is $p+1$-dimensional) to antisymmetric tensor fields of rank $p+1$. The gauge-invariant equations of motion for the antisymmetric part of $F$ are the usual ones $\partial_{M} F^{[M N]}=J^{N}$. For further details about equations of motion, the construction of Noether currents, continuity equation, etc... see [70] , [1].

It remains to be seen if this construction of C-space generalized Maxwell Electrodynamics of p-forms can be generalized to the Nonabelian case when we replace ordinary derivatives by gauge-covariant ones :

$$
F=d A \rightarrow F=D A=(d A+A \bullet A) .
$$

For example, one could define the graded-symmetric product $E_{M} \bullet E_{N}$ based on the graded commutator of Superalgebras:

$$
[A, B]=A B-(-1)^{s_{A} s_{B}} B A .
$$

$s_{A}, s_{B}$ is the grade of $A$ and $B$ respectively. For bosons the grade is even and for fermions is odd. In this fashion the graded commutator captures both the anti-commutator of two fermions and the commutator of two bosons in one stroke. Using ( 2-31 ) one may extend this definition to :

$$
E_{M} \bullet E_{N}=E_{M} E_{N}-(-1)^{s_{M} s_{N}} E_{N} E_{M} .
$$


$s_{M}, s_{N}$ is the grade of $E_{M}$ and $E_{N}$ respectively. Even or odd depending on the grade of the basis elements.

The task then is to decompose the C-space Maxwell action (6-8) which has a very rich structure into suitable field components, including the extraction of the spinorial pieces, and recover Smith's action after a truncation of a large number of terms.

One may generalize Maxwells theory to that of Born-Infeld nonlinear Electrodynamics in C-spaces based on our construction presented in this letter. Finally, the extension from a flat C-space to a curved C-space also warrants further investigation in order to couple C-space Yang-Mills theory to C-space gravity ( a higher derivative gravity with torsion [1] ) . This will be left for a future publication.

\subsection{Geometric Actions in Clifford-Phase Spaces}

\section{- On the relation between the curvature of $C$-space and the curvature of an} ordinary space

Let us now consider the $C$-space ( Clifford spaces ) curvature defined in [1] associated with a C-space interval $d \Sigma^{2}=G_{A B}(X) d X^{A} d X^{B}$. The indices $A, B$, can be of vector, bivector, etc., type. There are a series of special conditions that allows a simplification of the geometrical expressions for the C-space curvature components. It is instructive to consider a particular example associated with the polyvector indices : $A=[\mu \nu], B=[\alpha \beta]$, $C=\gamma, D=\delta$

$$
\left[\frac{\partial}{\partial_{x^{\mu_{1} \mu_{2}}}}, \frac{\partial}{\partial_{x^{\nu_{1} \nu_{2}}}}\right] \gamma_{\rho} \bullet \gamma_{\tau}=\mathcal{R}_{\left[\mu_{1} \mu_{2}\right]\left[\nu_{1} \nu_{2}\right] \rho \tau} .
$$

We can proceed in analogous way to calculate the other components of $\mathcal{R}_{A B C D}$ such as

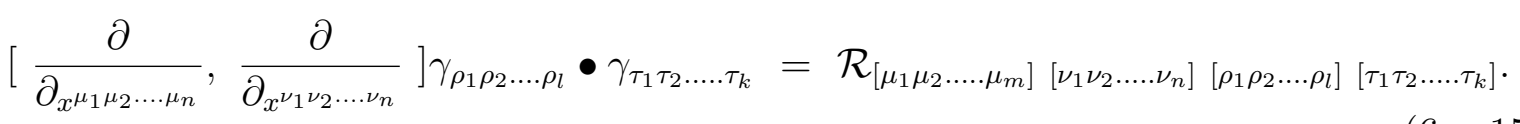

These contain higher powers of the curvature in an ordinary space. After performing the contractions and the corresponding higher order relations we obtain the expansion of the form

$$
\mathcal{R}=R+\alpha_{1} R^{2}+\alpha_{2} R_{\mu \nu} R^{\mu \nu}+\ldots+\operatorname{TORSION}
$$

where one must include the adequate powers of the Planck scale $L_{P}$ in the expansion to accomodate for the different dimensions of the quantities in the expansion. There are non-trivial Torsion terms as well since C-space Geometry has non-trivial Torsion.

So we have shown [1] that the $C$-space scalar curvature can be expressed as the sum of the products of the ordinary spacetime curvature. This bears a resemblance to the string effective action in curved spacetimes given by sums of powers of the curvature tensors based on the quantization of non-linear sigma models . For a nice review of strings in curved backgrounds and p-branes ( extended objects or extendons ) see [68]. 
If one sets aside the algebraic convergence problems when working with Clifford algebras in infinite dimensions, one can consider the possibility of studying Quantum Gravity in a very large number of dimensions which has been revisited recently [77] in connection to a perturbative renormalizable quantum theory of gravity in infinite dimensions. As we said earlier, Smith [32] has argued that infinite-dim Clifford algebras can be seen as tensor products of an infinite number of $\mathrm{Cl}(8)$ pieces that are roughly speaking, related to von Neumann $I I 1$ factors. Another interesting possibility is that an infinite series expansion of the powers of the scalar curvature could yield the recently proposed modified Lagrangians $R+1 / R$ of gravity to accomodate the cosmological accelerated expansion of the Universe [76], after a judicious choice of the algebraic coefficients is taken.

One may notice also that having a vanishing cosmological constant in C-space, $\mathcal{R}=$ $\Lambda=0$ does not necessarily imply that one has a vanishing cosmological constant in ordinary spacetime. For example, in the very special case of homogeneous symmetric spacetimes, like spheres and hyperboloids ( de Sitter, anti de Sitter ), where all the curvature tensors are proportional to suitable combinations of the metric tensor times the scalar curvature, it is possible to envision that the net combination of the sum of all the powers of the curvature tensors may cancel-out giving an overall zero value $\mathcal{R}=0$ despite the fact that the individual ordinary spacetime curvatures terms in the expansion may not be necessarily zero.

Thus a zero cosmological constant in C-space may not necessarily imply a zero cosmological constant in ordinary spacetime. This possibility deserves further investigation in relation to the cosmological constant problem. Of course, we must not forget the arguments exposed earlier about a lower and upper scale in Nature consistent with the Extended Relativity Theory in Clifford-Phase Spaces within the context of Nottale's Scale Relativity Theory. Nottale's proposal for the resolution of the cosmological constant problem is that it does not make sense to compare the vacuum energy at the Planck scale with the vaccum energy measured at Hubble scale, without taking into account the necessary Scale-Relativistic corrections : $\rho($ Planck $) / \rho($ Hubble $)=\left(R_{H} / L_{P}\right)^{2} \sim 10^{122}$ that account for such huge discrepancy in many orders of magnitude.

\section{- Curvature Scalar of the Tangent/Cotangent Bundle of Spacetime}

A natural Geometric action is provided by the scalar curvature $\mathcal{R}$ of the $8 D$ cotangent bundle. Brandt [12] has studied for some time the spacetime tangent bundle geometries from several perspectives, in particular within the framework of Finsler geometries. Given a tangent bundle metric

$$
d s^{2}=G_{M N} d x^{M} d x^{N}=g_{\mu \nu} d x^{\mu} d x^{\nu}+\rho^{2} g_{\mu \nu}\left(d v^{\mu}+\Gamma_{\alpha \beta}^{\mu} v^{\alpha} d x^{\beta}\right)\left(d v^{\nu}+\Gamma_{\gamma \delta}^{\nu} v^{\gamma} d x^{\delta}\right) \geq 0 .(6-17)
$$

in terms of the coordinates $x^{\mu}$ of the base manifold and the velocity $v^{\mu}$ variables of the tangent space manifold. $\rho$ is related to the maximal proper acceleration $a=c^{2} / \rho$. The scalar curvature of the $8 D$ tangent bundle manifold is [12]

$$
R^{(8)}=R^{(4)}+R^{(v)}-\frac{1}{4} F^{\alpha \beta \gamma} F_{\alpha \beta \gamma}-\frac{1}{4} T^{(\alpha \beta \gamma)} T_{(\alpha \beta \gamma)}+\ldots
$$




$$
-\frac{1}{4} \Pi^{(\alpha \beta \gamma)} \Pi_{(\alpha \beta \gamma)}+\ldots
$$

where $R^{(4)}$ is the $4 D$ spacetime scalar curvature. $R^{(v)}$ is the scalar curvature of the $4 D$ fiber manifold defined on the four-velocity space and in terms of $\Pi_{\alpha \beta}^{\mu}$, the Christoffel symbols defined on the four-velocity tangent space. The gauge potential is defined $A_{\nu}^{\mu}=$ $\rho v^{\lambda} \Gamma_{\lambda \nu}^{\mu}$, the gauge curvature field

$$
F_{\mu \nu}^{\alpha}=\left(\frac{\partial}{\partial_{\mu}}-\frac{1}{\rho} A_{\mu}^{\beta} \frac{\partial}{\partial v^{\beta}}\right) A_{\nu}^{\alpha}-\left(\frac{\partial}{\partial_{\nu}}-\frac{1}{\rho} A_{\nu}^{\beta} \frac{\partial}{\partial v^{\beta}}\right) A_{\mu}^{\alpha}
$$

The field $T_{\mu \nu}^{\beta}$ is defined in terms of the spacetime Christoffel symbol $\left\{\begin{array}{l}\beta \\ \mu \nu\end{array}\right\}$ as :

$$
T_{\mu \nu}^{\beta}=\left\{{ }_{\mu \nu}^{\beta}\right\}-\frac{1}{\rho} \frac{\partial}{\partial v^{\nu}} A_{\mu}^{\beta} .
$$

A vanishing gauge curvature field amounts to vanishing torsion and is also the condition for the Finsler spacetime tangent bundle to be compatible with Cartan's theory of Finsler spaces, to be complex and Kahler . For further details we refer to [12] .

An Einstein-Hilbert type of actions in the $8 D$ spacetime tangent bundle can be written as :

$$
\int_{V_{8}} \mathcal{D}^{4} x \mathcal{D}^{4} v \sqrt{G} R^{(8)}
$$

in terms of an appropriate tangent bundle measure. A variation of the action yields the analog of Einstein's vacuum field equations. The important feature is that in the infinite acceleration limit, and for the case of a Riemannian spacetime, the vacuum field equations reduce to the ordinary ones since the action then collapses to the ordinary Einstein-Hilbert action times a measure volume factor.

These sort of actions based on the $8 D$ spacetime tangent bundle geometry (Finsler like ) can be generalized to the spacetime cotangent-bundle in terms of the Phase-Space variables $x^{\mu}, p^{\mu}$ and furthermore to Clifford-Phase spaces with Clifford-valued $X, P$ polyvector coordinates. The latter action featuring the Clifford algebraic symmetries will be the higher derivatives version of the spacetime tangent bundle actions obtained in ( 6-18) . Notice thart these Clifford-Phase Space actions, after a spacetime/fiber decomposition, generate the desired gauge curvature field terms FF studied by Smith [32]. Vacaru over many years [27] has studied Finsler geometries and introduced the concept of a nonlinear connection which induces non-holonomic and anisotropic structures that are related to far more general metrics ( geometries ) than the ones studied in the past. In particular, by incorporating Clifford structures, (Dirac operator) Vacaru has developed Noncommutative Riemannian-Finsler geometries.

We shall discuss next yet another look at actions of the type $R^{(8)}$ ( 6-18) in relationship to the role of the diffeomorphisms ( diffs ) group and Holography.

\subsection{Gravity as Gauge Theories of Diffs and Holograpy}

The most general $4+4$ decomposition of the $8 D$ scalar curvature Geometric action was provided by [59]. Their main result is that $m+n$-dim Einstein gravity can be identified 
with an $m$-dimensional generally invariant gauge theory of $\operatorname{Diffs} N$, where $N$ is an $n$-dim manifold. Locally the $m+n$-dim space can be written as $\Sigma=\mathcal{M} \times \mathcal{N}$ and the metric $g_{A B}$ decomposes :

$$
\begin{gathered}
g_{\mu \nu}=\gamma_{\mu \nu}(x, y)+e^{2} \phi_{a b}(x, y) A_{\mu}^{a}(x, y) A_{\nu}^{b}(x, y) . \\
g_{\mu b}=g_{b \mu}=e A_{\mu}^{a}(x, y) \phi_{a b}(x, y) . \\
g_{a b}=\phi_{a b}(x, y) .
\end{gathered}
$$

where $e$ is the gauge coupling constant. This decomposition must not be confused with the Kaluza-Klein reduction where one imposes an isometry restriction on the $g_{A B}$ that turns $A_{\mu}^{a}$ into a gauge connection associated with the gauge group $G$ generated by isometry. Dropping the isometry restrictions allows all the fields to depend on all the coordinates $x, y$. Nevertheless $A_{\mu}^{a}(x, y)$ can still be identified as a connection associated with the infinite-dim gauge group of $\operatorname{Diffs} N$. The gauge transformations are now given in terms of Lie-brackets and Lie derivatives :

$$
\begin{gathered}
\delta A_{\mu}^{a}=-\frac{1}{e} D_{\mu} \xi^{a}=-\frac{1}{e}\left(\partial_{\mu} \xi^{a}-e\left[A_{\mu}, \xi\right]^{a}\right)=-\frac{1}{e}\left(\partial_{\mu}-e \mathcal{L}_{A_{\mu}}\right) \xi^{a} \\
A_{\mu} \equiv A_{\mu}^{a} \partial_{a} . \\
\mathcal{L}_{A_{\mu}} \xi^{a} \equiv\left[A_{\mu}, \xi\right]^{a} \\
\delta \phi_{a b}=-[\xi, \phi]_{a b}=\xi^{c} \partial_{c} \phi_{a b}+\phi_{a c} \partial_{b} \xi^{c}+\phi_{c b} \partial_{a} \xi^{c} . \\
\delta \gamma_{\mu \nu}=-\left[\xi, \gamma_{\mu \nu}\right] .
\end{gathered}
$$

Using eq-(6-22) the authors [59] have shown that the curvature scalar $R^{(m+n)}$ in $m+n$-dim decomposes into :

$$
\begin{gathered}
R^{(m+n)}=\gamma^{\mu \nu} R_{\mu \nu}^{(m)}+\frac{e^{2}}{4} \phi_{a b} F_{\mu \nu}^{a} F_{\rho \tau}^{b} \gamma^{\mu \rho} \gamma^{\nu \tau}+ \\
\phi^{a b} R_{a b}^{(n)}+\frac{1}{4} \gamma^{\mu \nu} \phi^{a b} \phi^{c d} D_{\mu} \phi_{a b} D_{\nu} \phi_{c d}+ \\
\frac{1}{4} \phi^{a b} \gamma^{\mu \nu} \gamma^{\rho \tau}\left[\partial_{a} \gamma_{\mu \rho} \partial_{b} \gamma_{\nu \tau}-\partial_{a} \gamma_{\mu \nu} \partial_{b} \gamma_{\rho \tau}\right] .
\end{gathered}
$$

plus total derivative terms given by

$$
\partial_{\mu}\left(\sqrt{-\gamma} \sqrt{\phi} J^{\mu}\right)-\partial_{a}\left(\sqrt{-\gamma} \sqrt{\phi} e A_{\mu}^{a} J^{\mu}\right)+\partial_{a}\left(\sqrt{-\gamma} \sqrt{\phi} J^{a}\right)
$$

with the currents:

$$
J^{\mu}=\gamma^{\mu \nu} \phi^{a b} D_{\nu} \phi_{a b}
$$




$$
J^{a}=\phi^{a b} \gamma^{\mu \nu} \partial_{b} \gamma_{\mu \nu}
$$

Therefore, Einstein gravity in $m+n$-dim describes an $m$-dim generally invariant field theory under the gauge transformations or Diffs $\mathcal{N}$. Notice how $A_{\mu}^{a}$ couples to the graviton $\gamma_{\mu \nu}$, meaning that the graviton is charged /gauged in this theory and also to the $\phi_{a b}$ fields. The " metric " $\phi_{a b}$ on $N$ can be identified as a non-linear sigma field whose self interaction potential term is given by : $\phi^{a b} R_{a b}^{(n)}$. The currents $J^{\mu}, J^{a}$ are functions of $\gamma_{\mu \nu}, A_{\mu}, \phi_{a b}$. Their contribution to the action is essential when there are boundaries involved; i.e. like in the $A d S / C F T$ correspondence.

When the internal manifold $\mathcal{N}$ is a homogeneous compact space one can perform a harmonic expansion of the fields w.r.t the internal $y$ coordinates, and after integrating w.r.t these coordinates $y$, one will generate an infinite-component field theory on the $m$ dimensional space. A reduction of the Diffs $\mathcal{N}$, via the inner automorphims of a subgroup $G$ of the Diffs $\mathcal{N}$, yields the usual Einstein-Yang-Mills theory interacting with a nonlinear sigma field. But in general, the theory described in (6-24) is by far richer than the latter theory. A crucial fact of the decomposition (6-24) is that each single term in (6-24) is by itself independently invariant under Diffs $\mathcal{N}$.

The second term, for example,

$$
\frac{1}{16 \pi G} \sqrt{\left|\operatorname{det}\left(\gamma_{\mu \nu}\right)\right|} \sqrt{\left|\operatorname{det}\left(\phi_{a b}\right)\right|} \times \frac{e^{2}}{4} \phi_{a b} F_{\mu \nu}^{a} F_{\rho \tau}^{b} \gamma^{\mu \rho} \gamma^{\nu \tau}
$$

is precisely the one that is be related to the large $N$ limit of $S U(N)$ YM [64].

The decomposition of the higer-dim Einstein-Hilbert action required to use a nonholonomic basis of derivatives $\partial_{\mu}-e A_{\mu}^{a} \partial_{a}$ and $\partial_{a}$ that allows a diagonal decomposition of metric and simplifies the computation of all the geometrical quantities. In this fashion the lower $m$-dimensional spacetime gauged " Ricci scalar" term $\gamma^{\mu \nu}(x, y) R_{\mu \nu}^{(m)}(x, y)$ and the internal space " Ricci scalar " term $\phi^{a b}(x, y) R_{a b}^{(n)}(x, y)$ are obtained. In the special case when $\gamma_{\mu \nu}(x)$ depends solely on $x$ and $\phi_{a b}(y)$ depends on $y$ then the spacetime gauged "Ricci scalar" coincides with the ordinary Ricci scalar $\gamma^{\mu \nu}(x) R_{\mu \nu}^{(m)}(x)$ and the internal space" Ricci" scalar becomes the true Ricci scalar of the internal space.

We have shown [67] that in this particular case the $D=m+n$ dimensional gravitational action restricted to $A d S_{m} \times S^{n}$ backgrounds admits a holographic reduction to a lower $d=m$-dimensional Yang-Mills-like gauge theory of diffs of $S^{n}$, interacting with a charged/gauged nonlinear sigma model plus boundary terms, by a simple tuning of the radius of $S^{n}$ and the size of the throat of the $A d S_{m}$ space. Namely, in the case of $A d S_{5} \times S^{5}$, the holographic reduction occurs if, and only if, the size of the $A d S_{5}$ throat coincides precisely with the radius of $S^{5}$ ensuring a precise cancellation of the scalar curvatures $\gamma^{\mu \nu} R_{\mu \nu}^{(m)}$ and $\phi^{a b} R_{a b}^{(n)}$ in eq- (6-24) leaving only the $\phi F F+\ldots$ terms [67].

The previous argument can also be generalized to gravitational actions restricted to de Sitter spaces, like $d S_{m} \times H^{n}$ backgrounds as well, where $H^{n}$ is an internal hyperbolic noncompact space of constant negative curvarture, and $d S_{m}$ is a de Sitter space of positive constant scalar curvature. The decomposition of the higher-dim scalar curvature provided a very straightforward explanation of why $A d S$ spaces played a crucial importance in the Maldacena's [66] AdS/CFT duality conjecture because the algebra of area-preserving diffs 
of the sphere is isomorphic to the large $N$ (basis dependent) limit of $S U(N)$ as shown by Hoppe long ago [65]. Recently there has been an argument against the validity of the isomorphism of the area-preserving diffs algebra of the sphere with the large $N$ limit ( basis dependent limit ) of $S U(N)$ [?].

Nevertheless, we have seen higher-dim gravity admits a holographic reduction to a lower-dim Yang-Mills like theory of diffs of the internal space. It is unfortunate that the important work of [59] that contained already the seeds of the holographic principle was largely ignored by the physics community.

Performing a mode expansion w.r.t the two internal variables $y^{1}, y^{2}$, and integrating afterwards w.r.t these variables yields an effective $4 D$ spacetime action with an infinite number of field-components. In particular, when the internal space is a circle, 4-dim Gauge theories based on the Virasoro algebra, diffs of a circle, have been constructed by [60] and 4-dim gauge theories based on the $w_{\infty}$ (area-preserving diffs of the plane) algebras have been constructed by [61] using the Feigin-Fuks-Kaplansky representation of $w_{\infty}$ algebras. Higgs matter fields in the adjoint representation were introduced also with the typical quartic potential terms which generated an infinte tower of massive spin 2 fields (massive higher spin fields in the case of $w_{\infty}$ gauge theories) after an sponteaneous symmetry breaking. These gauge theories based on the infinite-dim Virasoro and $w_{\infty}, w_{\infty}^{\infty}$ algebras are essential ingredients to understand further what is $W_{\infty}$ Geometry [62], [72]. The importance of non-critical $W_{\infty}$ strings within the context of Vasilev's higher spin theories [63] and ( super ) membranes in $D=11,27$ dimensions, Moyal deformations of gravity, the higher-dim Quantum Hall Effect, Chern-Simons Branes ( High-dimensional version of Knots ) and Topological Chern-Simons Matrix models was analyzed in [71] .

\section{An Einstein-Hilbert Action in Clifford-Phase Spaces : towards a Clifford Geometric Unification of all Forces}

To finalize we shall outline the most salient reasons why the Extended Relativity Theory in Clifford-Phase Spaces with a lower and upper length scales is a very promising path towards a Clifford Geometric Unification of all forces.

- We retain the $4 D$ spacetime. Despite that the Phase Space is $8 D$ one is dealing with an underlying $4 D$ spacetime with four coordinates $x^{\mu}$. The momenta coordinates $p^{\mu}$ can be seen as the dual variables implementing Born's Dual Relativity principle among coordinates and momenta which lead us to the construction of a Clifford-Phase Space Relativity with an upper and lower length scales so automatically it avoids the problems associated with ultraviolet ( short distance ) and infrared ( large distance) divergences in QFT.

- Clifford Algebras contain Spinors. There is no need to introduce Supersymmetry and Supergravity since Clifford algebras already contain spinors as right/left ideal elemnts of the Clifford Algebra. The Clifford Geometric calculus reproduces the Grassmannian and Berezin calculus [79] 
- No need for $D=11$ Supergravity .... The Clifford algebra $C l(1,7)$ is $2^{8}=256=$ $128+128$ dimensional which matches the 128 bosonic and 128 fermionic on-shell degrees of freedom of the $D=11$ Supergravity. The number of transverse and traceless degrees of freedom of the metric $g_{M N}$ in $D=11$ is $(9 \times 10 / 2)-1=44$. The antisymmeric tensor $A_{\mu \nu \rho}$ has $9 \times 8 \times 7 / 6=84$, so $44+84=128$ that match the degrees of freedom of the spin $3 / 2$ gravitino ( after imposing the vanishing of the gamma trace ).

- Emergence of $E_{8}, E_{7}, E_{6}, F_{4}, G_{2} \ldots$ We have explained earlier the arguments by Smith [32] why the Exceptional Lie Algebra $E_{8}, \ldots$ are encoded in the tensor products of $C l(8) \times$ $\mathrm{Cl}(8)$ by exploiting the modulo 8 real periodicity.

- The Clifford Geometric Unified Action. The Clifford Geometric Unified Action of all Forces is based on an Einstein-Hilbert action defined in the Clifford-Phase Space associated with the underlying $8 D$ Phase Space :

$$
\frac{1}{16 \pi \mathcal{G}_{N}} \int[\mathcal{D} \mathbf{Z}] \sqrt{\mathbf{G}} \mathbf{R} .
$$

where $\mathcal{D} \mathbf{Z}$ is the measure in the Clifford-Phase Space

$$
\mathcal{D} \mathbf{Z}=d \Omega d \tilde{\Omega}\left(d^{4} x\right)\left(d^{4} p\right)\left(d x^{\mu \nu}\right)\left(d p^{\mu \nu}\right) \ldots \ldots
$$

of $\operatorname{dim}_{R} C l(2,8)=2^{8}=256=16 \times 16$ effective dimensions. $\mathcal{G}_{N}$ is the analog of the Newtonian gravitational coupling that can be written as $L_{P}^{(256-2)}=L_{P}^{254}$ based on the four-dim Planck scale $L_{P}=\sqrt{G}_{N}$ in natural units of $\hbar=c=1$. The units of the scalar curvature $\mathbf{R}$ are (length) $)^{-2}$. $\mathbf{G}$ is the analog of the determinant of the CliffordPhase Space metric $\mathbf{G}_{M N}(\mathbf{X})$. $\mathbf{R}$ is the scalar curvature in the Clifford-Phase-Space associated with the $8 D$ phase space. As explained above, this scalar curvature admits a decomposition into a base manifold scalar curvature, a fiber manifold scalar curvature and a gauge field FF curvature term (plus additional terms). And all of these terms, in turn, admit a higher derivative expansion in powers of the ordinary spacetime, fiber manifold and gauge field curvatures. At low energies ( large distances ) there is a truncation (as we explained at the begining of section 4) where the holographic coordinates and holographic momenta decouple leading to Smith's $8 D$ action [32] (plus extra terms ) associated with point-particle degrees of freedom which reproduces the Standard Model and the ordinary Einstein-Hilbert action.

- Large number of degrees of freedom For example, the C-space Maxwell field $A=$ $A^{M} E_{M}$ (a Clifford polyvector in $D=8$ ) has $2^{8}=256$ components, and each single one of those 256 components $A=A^{M}(X) E_{M}$ depends on the polyvector coordinates $X=X^{M} E_{M}$ with 256 components. If we concentrate solely in the zero mode sector of $A(X)$ one has then $256 \times 256=2^{8} \times 2^{8}$ degrees of freedom that match precisely the $\operatorname{dim}_{R} C l(16)=\operatorname{dim}_{R}[C l(8) \otimes C l(8)]$ due to modulo 8 real periodicty. Once again, we have made contact with the $C l(16)$ algebra that contains the exceptional $E_{8} . E_{7}, E_{6}, F_{4}, G_{2} \ldots$ algebras as explained by Smith [32]. Similar study can be made with the net number of degrees of freedom of the zero mode sector of the scalar curvature in $C$-spaces.

- The large $N$ limit and Branes as Moyal deformations of Yang-Mills theories. The phase space coordinates can also be interpreted as the world manifold coordinates of extended objects. In [64] we have shown why p-brane actions can be derived from Moyal 
deformations of $S U(N)$ Yang-Mills theories and the classical limit $\hbar=0$ limit is related to the large $N$ limit.

- Quantization. A discussion of a Noncommutative QM in C-spaces was given in [29]. The role of Clifford algebras in Hopf algebras and Quantum Groups has been studied Majid [75] who has also emphasized the importance of Phase Space in defining a cogravity theory. Since the literature on Quantum Groups is so vast we refer to Majid's book for references.

\section{Acknowledgments}

We are indebted to M. Bowers for assistance. To Frank ( Tony ) Smith for many discussions and crucial explanations of his work and to Matti Pitkannen.

\section{References}

[1] C. Castro, M. Pavsic, " The Extended Relativity Theory in Clifford Spaces" commissioned review to appear in the Int. Jour . Mod. Phys. D ( 2004 ) .

[2] L. Nottale, La Relativite dans tous ses Etats. Hachette Lit. Paris 1999. L. Nottale, Fractal Spacetime and Microphysics, Towards Scale Relativity. World Scientific. Singapore, 1992.

[3] L. Nottale, "The Pioneer Anomalous Acceleration : a measurement of the cosmological constant at the Scale of the Solar system " [ arXiv : gr-qc/0307042 ] . Scale Relativistic Cosmology" Chaos, Solitons and Fractals 16 ( 2003 ) 539.

[4] P. Lounesto, Spinor Valued Regular Functions in Hypercomplex Analysis (ReportHTKK-MAT-A154 (1979) Helsinki University of Technology)

[5] B.G. Sidharth, Chaos, Solitons and Fractals 12 ( 2001 ) 1101.

[6] D. V Ahluwalia and C. Burgard, General Relativity and Gravitation 28 (10) (1996) 1163. D.V. Ahluwalia: General Relativity and Gravitation 29 (12) (1997) 1491. D.V. Ahluwalia, Phys. Letts A 275 (2000) 31. G.Adunas, E. Rodriguez-Milla and D.V. Ahluwalia, Phys. Letts B 485 (2000) 215.

[7] M. Born: Proc. Royal Society A 165 (1938) 291. Rev. Mod. Physics 21 (1949) 463.

[8] E. Caianiello, "Is there a maximal acceleration?", Lett. Nuovo Cimento 32 (1981) 65.

[9] V. Nesterenko, Class. Quant. Grav. 9 (1992) 1101; Phys. Lett. B 327 (1994) 50;

[10] V. Bozza, A. Feoli, G. Lambiase, G. Papini and G. Scarpetta, Phys. Let A 283 (2001) 53. V. Nesterenko, A. Feoli, G. Lambiase and G. Scarpetta, Phys. Rev D 60, 065001 (1999). 
[11] K. Rama, "Classical velocity in kappa-deformed Poincare algebra and amaximal acceleration" [arXiv: hep-th/0209129].

[12] H. Brandt: Contemporary Mathematics 196 (1996) 273. Chaos, Solitons and Fractals $10(2-3)(1999) 267$.

[13] F. Schuller, Annals of Phys. 299 (2002) 174.

[14] J. Lukierski, A. Nowicki, H. Ruegg, V. Tolstoy, Phys. Lett 264 (1991) 331. J. Lukierski, H. Ruegg, W. Zakrzewski: Ann. Phys 243 (1995) 90. J. Lukierski, A. Nowicki: Double Special Relativity verus kappa-deformed dynamics. [arXiv: hepth/0203065].

[15] J. Webb, M. Murphy, V. Flambaum, V. Dzuba, J. Barrow, C. Churchill, J. Prochaska, and A. Wolfe, " Further evidence for Cosmological Evolution of the Fine Structure Constant " Monthly Notices of the Royal Astronomical Society 327 (2001) 1208.

[16] J.P. Uzan, " The fundamental constants and their variations: observational status and theoretical motivations" [arXiv: hep-ph/0205340].

[17] G. Lambiase, G.Papini. G. Scarpetta: Maximal Acceleration Corrections to the Lamb Shift of one Electron Atoms [ arXiv: hep-th/9702130]. G. Lambiase, G.Papini. G. Scarpetta, Phys. Let A 224 (1998) 349. G. Papini," Shadows of a maximal acceleration" [arXiv: gr-qc/0211011].

[18] A. Feoli, G. Lambiase, G. Papini and G. Scarpetta, " Schwarzschild Field with Maximal Acceleration Corections" Phys. Lett A 263 ( 1999 ) 147. [ arXiv : grqc/9912089 ] . S. Capozziello, A. Feoli, G. Lambiase, G. Papini and G. Scarpetta, "Massive Scalar particles in a Modfied Schwarzschild Geometry ", Phys. Lett A 268 (2000) 247. [ arXiv : gr-qc/0003087 ] . V. Bozza , G. Lambiase, G. Papini and G. Scarpetta, "Quantum Violations of the Equivalence Principle in a Modfied Schwarzschild Geometry : Neutrino Oscillations. Maximal Acceleration Corections" [ arXiv : hep-ph/0012270 ] .

[19] C. Misner, K. Thorne and J. Wheeler, Gravitation, Chapter 25 ( W. H. Freeman and Co. San Francisco, 1973 ).

[20] C. Castro, Int. J. Mod. Phys. A 18 (2003) 5445 [arXiv: hep-th/0210061]

[21] L. Castellani: Phys. Lett B 327 (1994) 22. Comm. Math. Phys 171 (1995) 383.

[22] G.Amelino-Camelia, Phys. Lett B 510 (2001) 255. Int. J. Mod. Phys D 11 (2002) 35. Int. J. Mod. Phys D 11 (2002) 1643.

[23] K. Greisen, Phys. Rev. Lett 16 (1966) 748. G.T. Zatsepin, V. Kurmin, Sov. Phys. JETP Lett 4 (1966) 78. 
[24] M. Kafatos, S. Roy, and R. Amoroso, "Scaling in Cosmology and the Arrow of Time" Studies in the Structure of Time eds Buccheri et al ( Kluwer Academic, Plenum Publishers, New York, 2000 ) pp. 191-200. J. Glanz, Science 282 ( 1998 ) 2156.

[25] M. Toller, "The Geometry of Maximal Acceleration" [ArXiv: hep-th/0312016]. Int. Jour. Theor. Physics 29 (1990) 963.

[26] S. Low: Jour. Phys A Math. Gen 35 (2002) 5711. J. Math. Phys. 38 ( 1997 ) 2197.

[27] S. Vacaru, " Non-holonomic Clifford-Structures and Noncommutative RiemannFinsler Geometry" [ arXiv : math.DG/0408121]. S. Vacaru and N Vicol, " Nonlinear Connections and Clifford Structures" [ arXiv : math.DG/0205190]. S. Vacaru, " (Non) Commutative Finsler Geometry from String/M Theory " [arXiv: hepth/0211068]. S. Vacaru, A. Nadejda, Int. J. Math. Math. Sci. 23 (2004) 1189-1233. S. Vacaru, "Clifford Structures and Spinors on Spaces with Local Anisotropy", Buletinul Academiei de Stiinte a Republicii Moldova, Fizica si Tehnica [Izvestia Academii Nauk Respubliky Moldova, fizica i tehnika], 3, 53-62 (1995), Matscinet: 98i:53022 S. Vacaru, "Superstrings in Higher Order Extensions of Finsler Superspaces", Nucl. Phys. B, 434 (1997) $590-656$.

[28] A. Ashtekar, C. Rovelli and L. Smolin, Phys. Rev. Lett 69 (1992) 237. C. Rovelli, "A dialog on quantum gravity" [arXiv: hep-th/0310077] L. Freidel, E. Livine and Carlo Rovelli, Class.Quant.Grav. 20 (2003) 1463-1478. L. Smolin," How far are we from the quantum theory of gravity? " [ arXiv:hep-th/0303185]

[29] C.Castro, "On Noncommutative Yang's space-time algebra, Holography, Area Quantization and C-space Relativity" submitted to Class. Quan. Grav. CERNEXT-2004-090 preprint.

[30] C.N Yang, Phys. Rev 72 ( 1947 ) 874. Proceedings of the International Conference on Elementary Particles, ( 1965 ) Kyoto, pp. 322-323.

[31] S.Tanaka, Nuovo Cimento 114 B ( 1999 ) 49. S. Tanaka, " Noncommutative Field Theory on Yang's Space-Time Algebra, Covariant Moyal Star products and Matrix Model " [ arXiv : hep-th/0406166 ] . "Space-Time Quantization and Nonlocal Field Theory ..." [ arXiv : hep-th/0002001 ] . "Yang's Quantized Space-Time Algebra and Holographic Hypothesis " [ arXiv : hep-th/0303105] .

[32] F. Smith, Int. Jour. Theor. Phys. 24 ( 1985 ) 155. Int. Jour. Theor. Phys. 25 ( 1985 ) 355. "From Sets to Quarks" [ arXiv : hep-ph/9708379] and [ CERN CDS EXT2003-087 ]. G. Gonzalez-Martin, Physical Geometry University of Simon Bolivar, publishers, Caracas, June 2000, 265 pages, ISBN : 9800767495. G. Gonzalex-Martin, "The proton/electron Geometric Mass Ratio" [ arXiv : physics/0009052 ] . G. Gonzalez-Martin, "The fine structure constant from Relativistic Groups" [ arXiv : physics/0009051 ] 
[33] C. Beck, Spatio-Temporal Vacuum Fluctuations of Quantized Fields Advances in Nonlinear Dynamics vol. 21 ( World Scientific, Singapore 2000 ) . "Chaotic Strings and Standard Model Parameters" Physica 171 D ( 2002 ) 72. [arXiv : hepth/0105152].

[34] V. Vladimorov, I. Volovich, I . Zelenov, p-adic Numbers in Mathematical Physics ( World Scientific, Singapore, 1994 ) .

L. Brekke, P. Freund, Phys. Reports 231 ( 1993 ) 1.

[35] M. Pitkannen, " Topological Geometrodynamics I, II " Chaos, Solitons and Fractals 13 , no. 6 ( 2002 ) 1205 and 1217.

[36] P. Noyes, Bit-String Physics : A discrete and finite approach to Natural Philosophy eds J. C van der Berg, Series of Knots in Physics vol. 27 ( World Scientific, Singapore 2001 ).

[37] L. Smolin, J. Kowalksi-Glikman, " Triply Special Relativity " [ arXiv : hepth/0406276] . S.S. McGaugh, " Modfied Newtonian Dynamics as an alternative to Dark matter" [ arXiv ; astro-ph/0204521] . A. Aguirre, " Alternatives to Dark Matter" [ arXiv : astro-ph/0305572] .

[38] L. Nam Chang, " Some Consequences of the Hypothesis of Minimal Lengths" [ arXiv : hep-th/0405059 ] .

[39] C. Castro, Foundations of Physics 8 ( 2000 ) 1301.

[40] C. Castro, Foundations of Physics 34 , no. 7 ( 2004) 107.

[41] J. Armenta, J. A. Nieto," The de Sitter Relativistic Top Theory" [arXiv : 0405254]

[42] R. Vilela Mendes, J. Phys. A 27 ( 1994 ) 8091-8104. R. Vilela-Mendes, " Some consequences of a noncommutative space-time structure " [ arXiv : hep-th/0406013] C. Chryssomalakos, E. Okon, " Linear Form of 3-scale Special Relativity Algebra and The Relevance of Stability" [ arXiv : 0407080 ]

[43] Y. Nambu, Phys. Rev D 7 ( 1973 ) 2405.

[44] J. Moffat, " Quantun Gravity Momentum Representation an Maximum Invariant Energy " [ arXiv : gr-qc/0401117 ]

[45] D. Friedan, " Two Talks on a tentative theory of large distance physics" [ arXiv : hep-th/0212268 ] .

[46] D. Cole, "Contrasting Quantum Cosmologies: [ arXiv : gr-qc/0312045 ] . R. G . Vishwakarma," A Machian Model of Drak Energy" [ arXiv : gr-qc/0205075 ] . A. Buchalter, "On the time variations of $c, G$ and $h$ and the dynamics of the Cosmic expansion " [ arXiv : astro-ph/0403202 ] . 
[47] D. Roscoe, General Relativity and Gravitation 36 ( 2004 ) 3. Gen. Relativity and Gravitation 34 ( 2002 ) 577. Astrophysics and Space Science 285 no. 2 ( 2003 ) 459.

[48] D. Steer, M. Parry, Int. Jour of Theor. Physics 41 no. 11 ( 2002 ) 2255.

[49] J. F. Adams, Lectures on Exceptional Groups University of Chicago ( 1996 ).

[50] L. K. Hua, Harmonic Analysis of Functions of Several Complex Variables in the Classical Domain AMS ( 1963 ).

[51] J. Conway, N. Sloane, Sphere Packings, Lattices and Groups Springer Verlag ( 1993 ) .

[52] J. Lohmus, E. Paal and L. Sorgsepp, Nonassociative Algebras in Physics Hadronic Press ( 1994 ).

[53] G. Dixon, Division Algebras Kluwer ( 1994 )

[54] A. Sudbery J. Phys. A Math. Gen 17 ( 1984 ) 939-955.

[55] J, Baez, "The Octonions" [ arXiv : math.RA/0105155 ] . J.Baez http://math.ucr.edu/home/baez/week.175.html

[56] J. Lansberg, L. Manivel, [ math.AG/9810140 ] .

[57] J. Faraut, S. Kaneyuki, A. Koranyi, Q. Lu and G. Roos, Analysis and Geometry on Complex Homoeneous Domains Birkhauser ( 2000 ).

[58] T. Smith, " Golden Bars of Consensus and the Truth ( Top ) Quark" [ arXiv : physics/0006041] .

[59] Y. Cho, K. Soh and Q. Park, J. Yoon : Phys. Lets B 286 (1992) 251. J. Yoon, Phys. Letts B 308 ( 1993 ) 240; J. Yoon, Phys. Lett A 292 ( 2001 ) 166. J. Yoon, Class. Quan. Grav 16 ( 1999 ) 1863.

[60] Y. Cho, W. Zoh, Phys. Review D 46 ( 1992) 3483.

[61] W. Zhao, Jour. Math. Phys. 40 ( 1999 ) 4325.

[62] C. Hull, Phys. Letts B 269 ( 1991) 257.

[63] M. Vasiliev, " Higher Spin Gauge Theories, Star Product and AdS spaces " [ arXiv : hep-th/9910096] . M. Vasiliev , S. Prokushkin, "3D Higher-Spin Gauge Theories with Matter". [ arXiv : hep-th/9812242, hep-th/9806236] . 
[64] C. Castro, " Branes from Moyal Deformation quantization of Generalized YangMills " [ arXiv : hep-th/9908115]. S. Ansoldi, C. Castro, E. Spallucci, Phys. Lett B 504 (2001) 174. S. Ansoldi, C. Castro, E. Spallucci, Class. Quant. Grav. 18 (2001) L23. S. Ansoldi, C. Castro, E. Spallucci, Class. Quan. Gravity 18 (2001) L17-L23. S. Ansoldi, C. Castro, E. Guendelmann and E. Spallucci, Class. Quant. Gravity 19 (2002) L 135.

[65] J. Hoppe," Quantum Theory of a Relativistic Surface" Ph.D Thesis MIT (1982).

[66] J. Maldacena, Adv. Theor. Math. Phys 2 (1998) 231.

[67] C. Castro, Europhysics Letters 61 ( 4 ) (2003) 480-484. Class. Quant. Gravity 20 ( 16 ) ( 2003 ) 3577-3592.

[68] Y. Ne'eman, E. Eizenberg, "Membranes and Other Extendons ( $p$-branes)" World Scientific Lecture Notes in Physics vol. 391995.

[69] C. Castro, Foundations of Physics. 8 ( 2000 ) 1301

[70] C. Castro, Mod. Phys. Letts A 19 , no.1 ( 2004 ) 19-27.

[71] C. Castro, "Moyal deformations of Gravity via $S U(\infty)$ Gauge Theories , Branes and Topological Chern-Simons Matrix Models" to appear in General Relativity and Gravitation, 36 (2004 ).

[72] C. Castro, Journal of Geometry and Physics 33 (2000) 173-190

[73] M. Pavsic, The Landscape of Theoretical Physics : A Global View, from Point Particles to the Brane World and Beyond, in Search of a Unifying Principle Kluwer Academic Publishers ( 2001 ).

[74] R. Penrose, W. Rindler, Spinors and Spacetime Cambridge Monographs on Mathematical Physics, vols 1 and 2 , Cambridge University Press ( 1986 ).

[75] S. Majid, Quantum Groups Cambridge University Press

[76] Capozziello, S. Carloni and A. Troisi, " Quintessence without scalar fields " [arXiv: astro-ph/0303041]. S. Carroll, V. Duvvuri, M. Trodden and M. Turner, "Is Cosmic Speed-Up Due to New Gravitational Physics? " [arXiv: astro-ph/0306438]. A. Lue, R. Scoccimarro and G. Strakman, " Differentiating between Modified Gravity and Dark Energy" [arXiv: astro-ph/0307034].

[77] N. Bjerrus-Bohr, " Quantum Gravity at large number of dimensions" [arXiv: hepth/0310263]. J.F. Donoghue, Phys. Rev. D 50 (1994) 3874. A. Strominger, Phys. Rev. D 24 (1981) 3082.

[78] K. Avinash and V. I. Rvachev, Foundations of Physics 30(2000) 139. 
[79] A.Lasenby, C. Doran and S. Gull : Phil. Trans. Roy Soc 356 487. A.Lasenby, C. Doran and S. Gull : Jour. Math. Phys 39 ( 1998 ) 3303. Trayling and W. Baylis: J. Phys. A 34 (2001) 3309. J. Chisholm and R. Farwell : J. Phys. A 32 ( 1999 ) 2805. J. Chisholm and R. Farwell : Foundations of Physics 25 ( 1995 ) 1511. 